\title{
LIMIT THEOREMS FOR ISOTROPIC RANDOM WALKS ON TRIANGLE BUILDINGS
}

\author{
MARC LINDLBAUER and MICHAEL VOIT
}

(Received 18 December 2000; revised 23 August 2001)

\author{
Communicated by A. H. Dooley
}

\begin{abstract}
The spherical functions of triangle buildings can be described in terms of certain two-dimensional orthogonal polynomials on Steiner's hypocycloid which are closely related to Hall-Littlewood polynomials. They lead to a one-parameter family of two-dimensional polynomial hypergroups. In this paper we investigate isotropic random walks on the vertex sets of triangle buildings in terms of their projections to these hypergroups. We present strong laws of large numbers, a central limit theorem, and a local limit theorem; all these results are well-known for homogeneous trees. Proofs are based on moment functions on hypergroups and on explicit expansions of the hypergroup characters in terms of certain two-dimensional Tchebychev polynomials.
\end{abstract}

2000 Mathematics subject classification: primary 60B15; secondary 60F05, 60F $15,33 \mathrm{D} 52,20 \mathrm{E} 42$, $43 \mathrm{~A} 62$.

Keywords and phrases: triangle buildings, Hall-Littlewood polynomials, polynomial hypergroups, isotropic random walks, law of large numbers, central limit theorem, local limit theorem.

\section{1: Introduction}

In this paper we transfer some limit theorems on isotropic random walks on homogeneous trees to triangle buildings, that is, thick locally finite buildings of type $\tilde{A}_{2}$. To explain the results of this paper, we first recapitulate a method to analyze isotropic random walks on certain graphs; we then specialize this approach to homogeneous trees and triangle buildings.

Let $\Gamma$ be the vertex set of some locally finite, connected, and undirected graph, which carries the usual metric $d$. Assume there exists a closed subgroup $G$ of the automorphism group of $(\Gamma, d)$ (which carries the compact-open topology) such that $G$

(C) 2002 Australian Mathematical Society $1446-7887 / 2000 \$ A 2.00+0.00$ 
acts transitively on $\Gamma$. Then $G$ is a totally disconnected locally compact group, the stabilizer $H_{e} \subset G$ of any $e \in \Gamma$ is a compact open subgroup, and we may identify the discrete spaces $G / H_{e}=\left\{g H_{e}: g \in G\right\}$ and $\Gamma$. Moreover, the discrete orbit space $\Gamma^{H_{e}}:=\left\{H_{e}(x): x \in \Gamma\right\}$ may be identified with the discrete double coset space $G / / H_{e}:=\left\{H_{e} g H_{e}: g \in G\right\}$. Let $\omega_{H_{e}} \in M^{1}(G)$ be the normalized Haar measure of $H_{e}$. Then the space

$$
M_{b}\left(G \| H_{e}\right):=\left\{\mu \in M_{b}(G): \omega_{H_{e}} * \mu * \omega_{H_{e}}=\mu\right\}
$$

of all $H_{e}$-biinvariant bounded signed measures on $G$ is a Banach $*$-subalgebra of $M_{b}(G)$ with the convolution as product and the total variation norm as norm. Moreover, $M_{b}\left(G \| H_{e}\right)$ is isometrically isomorphic with the space $M_{b}\left(G / / H_{e}\right)$ of all bounded signed measures on $G / / H_{e} \simeq \Gamma^{H_{e}}$. Via this isomorphism, $M_{b}\left(G / / H_{e}\right)$ receives a canonical Banach $*$-algebra structure with a convolution which admits almost all properties of a group convolution. More precisely, $\left(G / / H_{e}, *\right)$ is a discrete hypergroup in the sense of Dunkl, Jewett, and Spector; for details on hypergroups see the monograph [BH]. In particular, the convolution on $M_{b}\left(G / / H_{e}\right)$ is probability preserving. The hypergroup structure on $G / / H_{e}$ can be used to analyze isotropic random walks on $\Gamma$. A homogeneous Markov chain $\left(X_{n}\right)_{n \geq 0}$ on $\Gamma$ starting at some vertex $e \in \Gamma$ will be called an isotropic random walk if

$$
\mathbf{P}\left(X_{n+1}=x \mid X_{n}=y\right)=\mathbf{P}\left(X_{n+1}=g(x) \mid X_{n}=g(y)\right)
$$

for all $x, y \in \Gamma, g \in G, n \geq 0$. As all $X_{n}$ are uniformly distributed on all $H_{e}$-orbits, no information on the distributions of $\left(X_{n}\right)_{n \geq 0}$ is lost under the projection

$$
\pi: \Gamma \simeq G / H_{e} \rightarrow G / / H_{e} \simeq \Gamma^{H_{e}} .
$$

More precisely, $\left(Y_{n}:=\pi\left(X_{n}\right)\right)_{n \geq 0}$ is a homogeneous Markov chain on $G / / H_{e}$, and there exists a unique probability measure $\mu \in M^{1}\left(G / / H_{e}\right)$ such that

$$
\mathbf{P}\left(Y_{n+1}=x \mid Y_{n}=y\right)=\mu * \delta_{y}(\{x\}) \text { for } x, y \in G / / H_{e}, n \geq 0,
$$

where $\delta_{y}$ denotes the point measure in $y$. In other words, $\left(Y_{n}\right)_{n \geq 0}$ is a random walk on the hypergroup $G / / H_{e}$. Clearly, $Y_{n}$ has the distribution $\mu^{n}$, the $n$th convolution power of $\mu$ with respect to $*$. If $G / / H_{e}$ admits 'sufficiently nice features', it will be possible to derive limit theorems for $\left(Y_{n}\right)_{n \geq 0}$, which can be regarded as limit theorems for $\left(X_{n}\right)_{n \geq 0}$. In particular, if the convolution on $G / / H_{e}$ is commutative (that is, $\left(G, H_{e}\right)$ is a Gelfand pair), then one can use the spherical Fourier transform (that is, the Fourier transform on the commutative hypergroup $\left.\left(G / / H_{e}, *\right)\right)$ to obtain limit theorems for $\left(Y_{n}\right)_{n \geq 0}$. This is in particular the case for the examples considered in this paper.

Assume now that $\Gamma$ is the vertex set of a homogeneous tree of valency $q+1$ with $q \geq 1$. Fix some $e \in \Gamma$. Then two vertices $x, y \in \Gamma$ are contained in the same $H_{e}$-orbit 
if and only if $d(x, e)=d(y, e)$ holds. We hence may identify $G / / H_{e} \simeq \Gamma^{H_{e}}$ with $\mathbb{N}_{0}=\{0,1, \ldots\}$. Moreover, simple counting shows that the hypergroup convolution of point measures on $\mathbb{N}_{0}$ is given by

$$
\delta_{m} * \delta_{n}=\frac{1}{(q+1) q^{m \wedge n-1}} \delta_{|m-n|}+\sum_{k=1}^{m \wedge n-1} \frac{q-1}{(q+1) q^{m \wedge n-k}} \delta_{|m-n|+2 k}+\frac{q}{q+1} \delta_{m+n}
$$

for $m, n \in \mathbb{N}$; see [V1]. Notice that $\delta_{m} * \delta_{n}$ are probability measures for all $m, n \in \mathbb{N}_{0}$ and $q \geq 1$. In particular, the convolution $*$ is commutative, and the spherical functions of $\Gamma$ can be described in terms of Bernstein-Szegö polynomials which are given by $C_{0}^{q}(x)=1$ and, for $n \in \mathbb{N}$,

$$
C_{1}^{q}(x)=\frac{2 \sqrt{q}}{q+1} x, \quad C_{1}^{q}(x) C_{n}^{q}(x)=\frac{q}{q+1} C_{n+1}^{q}(x)+\frac{1}{q+1} C_{n-1}^{q}(x) .
$$

These polynomials are orthogonal on $[-1,1]$ with respect to the weight function

$$
w_{q}(x):=\frac{\sqrt{1-x^{2}}}{x_{q}^{2}-x^{2}} \quad \text { with } \quad x_{q}:=\frac{q+1}{2 \sqrt{q}} \geq 1 ;
$$

for details see [AW, BH, V1]. Notice that for $q=1$ we have $\Gamma \simeq \mathbb{Z}$ and the $C_{n}^{1}$ are the Tchebychev polynomials of the first kind, that is, $C_{n}^{1}(\cos t)=T_{n}(\cos t):=\cos (n t)$. Moreover, (1.4) yields that

$$
C_{n}^{q}(x)=\frac{1}{(q+1) q^{n / 2-1}} \cdot\left(U_{n}(x)-\frac{1}{q} U_{n-2}(x)\right) \quad(n \geq 2, x \in \mathbb{R}),
$$

where the $U_{n}(\cos t):=\sin ((n+1) t) / \sin (t)$ are the Tchebychev polynomials of the second kind; see [AW, L2]. Most data of the commutative hypergroup $\left(\mathbb{N}_{0}, *\right)$ can now be determined in terms of $C_{n}^{q}$ : The dual space

$$
\widehat{\mathbb{N}}_{0}:=\left\{\alpha \in C_{b}\left(\mathbb{N}_{0}\right): \alpha \mathbb{R} \text {-valued and } \delta_{m} * \delta_{n}(\alpha)=\alpha(m) \alpha(n) \text { for } m, n \in \mathbb{N}_{0}\right\}
$$

$\left(C_{b}\left(\mathbb{N}_{0}\right)\right.$ being the space of all bounded $\mathbb{C}$-valued functions on $\left.\mathbb{N}_{0}\right)$ is

$$
\widehat{\mathbb{N}}_{0}=\left\{\alpha_{x}: x \in\left[-x_{q}, x_{q}\right], \alpha_{x}(n)=C_{n}^{q}(x) \text { for } n \in \mathbb{N}_{0}\right\},
$$

after identification of $\left[-x_{q}, x_{q}\right]$ and $\widehat{\mathbb{N}}_{0}$, the Plancherel measure is $\left.w_{q}(x) d \lambda\right|_{[-1,1\}}(x)$ with $\left.\lambda\right|_{[-1,1]}$ the Lebesgue measure on $[-1,1]$ and $w_{q}$ as in (1.5). Moreover, $x_{q}$ corresponds to the trivial character; for further details on polynomial hypergroups see $[\mathrm{BH}]$. One can now use Fourier analysis to derive limit theorems for random walks on $\left(\mathbb{N}_{0}, *\right)$. In particular, moment functions in the sense of Zeuner $[\mathrm{Z} 1, \mathrm{Z2}]$ were used in Voit [V1, V2, V3] to derive limit theorems for quite general polynomial hypergroups 
which cover the examples above. Similar limit theorems for homogeneous trees were given earlier by Sawyer [Sa]. Moreover, using asymptotic results for $C_{n}^{q}$ at $x_{q}$ based on (1.6), Lindlbauer [L2] gave a central limit theorem for random walks on $\left(\mathbb{N}_{0}, *\right)$ together with Berry-Esseen type rates of convergence.

In this paper we transfer the Fourier analytic approach above to triangle buildings. We do not need formal building theory here and refer to [B, CMSZ, R, T] for details. Informally, a triangle building $\Delta$ is a simplicial complex consisting of vertices, edges, and triangles, where any two triangles are connect by a gallery of triangles. The vertices and edges form a graph which is assumed to be locally finite. Let $\Gamma$ be the set of vertices; then each $v \in \Gamma$ has a type $\tau(v) \in\{0,1,2\}$ where each triangle has a vertex of each type. Thickness implies that each edge belongs to the same number of triangles. We denote this number by $q+1$ and call $q$ the order of $\Delta$. Unlike the tree case, $\Delta$ is not determined uniquely by $q$; see Tits [T].

Now take two vertices $u, v \in \Gamma$. Then there is a subcomplex $A$ of $\Delta$ with $u, v \in A$ such that $A$ is isomorphic to a euclidian plane tessellated regularly by equilateral triangles. Moreover, there are unique $m, n \in \mathbb{N}_{0}$ and rays $\left(u_{0}=u, u_{1}, \ldots, u_{n}\right)$ and $\left(u_{0}^{\prime}=u, u_{1}^{\prime}, \ldots, u_{m}^{\prime}\right)$ in $A$ with $d\left(u_{n}, v\right)=m, d\left(u_{m}^{\prime}, v\right)=n$, and with $\tau\left(u_{i+1}\right) \equiv$ $\tau\left(u_{i}\right)+1(\bmod 3)$ and $\tau\left(u_{i+1}^{\prime}\right) \equiv \tau\left(u_{i}^{\prime}\right)-1(\bmod 3)$ for $i \geq 0$. Furthermore, $m$ and $n$ are independent of $A$. Hence, for $u \in \Gamma$ and $m, n \in \mathbb{N}_{0}$, the set $S_{n, m}(u)$ consisting of all $v \in \Gamma$ with the properties above is well defined. Moreover, $v \in S_{n, m}(u)$ is equivalent to $u \in S_{m, n}(v)$. Let $G_{t r}$ be the group of all 'type-rotating' automorphisms of $\Gamma$, that means for each $g \in G_{t r}$ there is a $c_{g} \in\{0,1,2\}$ with $\tau(g(u)) \equiv \tau(u)+c_{g}$ (mod 3) for all $u \in \Gamma$. Then the stabilizer subgroup $H_{e}$ of any fixed vertex $e$ acts on each $S_{n, m}(e)$. If $G_{t r}$ acts transitively on $\Gamma$ and $H_{e}$ acts even transitively on each $S_{n, m}(e)$ (which is the case in the examples discussed below), then the results mentioned above imply that isotropic random walks on $\Gamma$ can be analyzed by considering their projections which live on the associated hypergroup structures on

$$
G_{i r} / / H_{e} \simeq\left\{S_{n, m}(e): n, m \in \mathbb{N}_{0}\right\} \simeq \mathbb{N}_{0}^{2} .
$$

In [CM], it was shown that, with no assumptions on $G_{t r}$ and $H_{e}$, any triangle building $\Delta$ gives rise to a hypergroup structure on $\mathbb{N}_{0}^{2}$ (the $A_{m, n}$ in $[C M]$ correspond to $(m, n) \in$ $\left.\mathbb{N}_{0}^{2}\right)$. In this context, an isotropic random walk on the vertices is defined to be one for which $\mathbf{P}\left(X_{n+1}=y \mid X_{n}=x\right)=: p_{n}(x, y)$ depends only on the 'coordinates' of $y$ with respect to $x$. That is, if $y \in S_{r, s}(x)$ and $v \in S_{r, s}(u)$, then $p_{n}(u, v)=p_{n}(x, y)$. It was checked in [CM, MZ] that these hypergroups are commutative, and their dual spaces, Haar measures, and Plancherel measures were determined. We use these results to derive limit theorems for random walks on these hypergroups. For this, we recapitulate several results of $[\mathrm{CM}, \mathrm{MZ}]$ in the next section in the language of hypergroups. In particular, we identify the hypergroup characters as orthogonal polynomials in two variables which are orthogonal with respect to certain measures on the compact region 
$Z_{1} \subset \mathbb{C}$ bounded by Steiner's hypocycloid. These polynomials are closely related to the Hall-Littlewood polynomials studied in [Mac2], and it follows from a result of Miller Maley [M] that the orthogonal polynomials above, which depend on the parameter $q$, actually generate polynomial hypergroups for all $q \in \mathbb{R}$ with $q \geq 1$. The results of this paper therefore hold for all $q \in \mathbb{R}$ with $q \geq 1$ (where the limit case $q=1$ has to be treated separately sometimes).

In Section 3 we introduce 2-dimensional Tchebychev polynomials of the first and second kind whose orthogonality measures are supported by $Z_{1}$. These polynomials are part of a more general class of ultraspherical polynomials studied by Koornwinder [Kw]. We present expansions of our hypergroup characters in terms of these polynomials. These expansions will be crucial for some asymptotic results which finally lead to a central limit theorem in Section 6. Proofs of this central limit theorem as well as of the strong laws of large numbers in Section 5 will be based in this paper on the concept of moment functions on hypergroups developed by Zeuner [Z1, Z2]. We study these moment functions in Section 4. In Section 5 we present a local central limit theorem for random walks on triangle buildings; a similar result for homogeneous trees can be found in [P]. We also mention a central limit theorem in [BG] for random walks on $\mathbb{N}_{0}^{2}$ associated with disk polynomials.

We finally present examples of triangle buildings from $[\mathrm{B}, \mathrm{R}]$ for illustration: Let $q$ be a prime power and $F$ a local field with residual field of order $q$ and valuation $v$. Let $\mathscr{O}:=\{x \in F: v(x) \geq 0\}$ and $\omega \in \mathscr{O}$ with $v(\omega)=1$. Let $L$ be a lattice in $V:=F^{3}$, that is, an $\mathscr{O}$-submodule of $V$ of the form $\left\{\sum_{i=1}^{3} a_{i} v_{i}: a_{i} \in \mathscr{O}\right\}$, where $\left\{v_{1}, v_{2}, v_{3}\right\}$ is an $F$-basis of $V$. Lattices $L, L^{\prime}$ are called equivalent if $L^{\prime}=t L$ for some $t \in F$. The associated equivalence classes $[L]$ will form the vertices of a building $\Delta_{F}$, where the triangles consist of distinct vertices $\left[L_{1}\right],\left[L_{2}\right],\left[L_{3}\right]$ satisfying $L_{1} \supset L_{2} \supset L_{3} \supset \omega L_{1}$. Then $G:=P G L(3, F)$ acts on $\Delta_{F}$ by left multiplication in a type-rotating way, and the stabilizer $H_{e}$ of any vertex $e \in \Gamma_{F}$ acts transitively on all $S_{n, m}(e)$; see [CM, Section 2]. Further examples of triangle buildings with prime power orders are given in [CMSZ]. It is a long-standing open problem whether examples exist for $q$ not a prime power.

A major part of this paper consists of the thesis [L1]. The research for this thesis was carried while the the first author held a research position at the GSFForschungszentrum für Umwelt und Gesundheit.

The authors would like to thank Don Cartwright and an anonymous referee for some valuable hints. Don Cartwright in particular informed us about the connection of the polynomials in this paper to the Hall-Littlewood polynomials, which leads to the positivity results in Remark 2.3. After submission of the paper, we learned about the paper [CW] which contains similar limit theorems for buildings of type $\tilde{A}_{n}$. 


\section{Polynomial hypergroups associated with triangle buildings}

Let $\Delta$ be a triangle building of order $q$. As explained in the introduction, this gives rise to a hypergroup $\left(\mathbb{N}_{0}^{2}, *\right)$ which has the following properties by $[\mathrm{CM}]$ :

PROPOSITION 2.1. The commutative hypergroup $\left(\mathbb{N}_{0}^{2}, *\right)$ has identity $(0,0)$ and involution $(m, n)^{-}=(n, m)$ for $m, n \in \mathbb{N}_{0}$. The Haar measure $\omega$ with $\omega(\{(0,0)\})=1$ is given by $\omega(\{(m, n)\})=N_{m, n}$ with

$$
\begin{aligned}
& N_{0,0}:=1, \quad N_{m, 0}:=N_{0, m}:=\left(q^{2}+q+1\right) q^{2(m-1)}, \\
& N_{m, n}:=\left(q^{2}+q+1\right)\left(q^{2}+q\right) q^{2(m+n-2)}: \quad(m, n \geq 1) .
\end{aligned}
$$

Moreover, the convolution of point measures on $\left(\mathbb{N}_{0}^{2}, *\right)$ satisfies

$$
\begin{aligned}
\delta_{(0, n)} * \delta_{(1,0)} & =\frac{1}{q^{2}+q+1}\left(\left(q^{2}+q\right) \delta_{(1, n)}+\delta_{(0, n-1)}\right) \\
\delta_{(m, 0)} * \delta_{(1,0)} & =\frac{1}{q^{2}+q+1}\left(q^{2} \delta_{(m+1,0)}+(q+1) \delta_{(m-1,1)}\right) \\
\delta_{(m, n)} * \delta_{(1,0)} & =\frac{1}{q^{2}+q+1}\left(q^{2} \delta_{(m+1, n)}+q \delta_{(m-1, n+1)}+\delta_{(m, n-1)}\right) \\
\delta_{(m, 0)} * \delta_{(0,1)} & =\frac{1}{q^{2}+q+1}\left(\left(q^{2}+q\right) \delta_{(m, 1)}+\delta_{(m-1,0)}\right) \\
\delta_{(0, n)} * \delta_{(0,1)} & =\frac{1}{q^{2}+q+1}\left(q^{2} \delta_{(0, n+1)}+(q+1) \delta_{(1, n-1)}\right) \\
\delta_{(m, n)} * \delta_{(0,1)} & =\frac{1}{q^{2}+q+1}\left(q^{2} \delta_{(m, n+1)}+q \delta_{(m+1, n-1)}+\delta_{(m-1, n)}\right)
\end{aligned}
$$

for $m, n \geq 1$.

Induction on $m+n$ shows that (2.1) determines the hypergroup convolution uniquely, and that the Banach algebra $M_{b}\left(\mathbb{N}_{0}^{2}\right)$ of all bounded measures on $\mathbb{N}_{0}^{2}$ is the closure of the subalgebra generated by $\delta_{(1,0)}$ and $\delta_{(0,1)}$. Therefore, multiplicative functions $f$ on $\left(\mathbb{N}_{0}^{2}, *\right)$, which satisfy

$$
\left(\delta_{(m, n)} * \delta_{(k, l)}\right)(f)=f(m, n) \cdot f(k, l) \text { for all } k, l, m, n \in \mathbb{N}_{0},
$$

are determined uniquely by their values at $(1,0)$ and $(0,1)$. It was in fact shown in [CM, MZ] that for each $z, w \in \mathbb{C}$, there exists a (unique) multiplicative function $f_{z . w}$ with

$$
f_{z, w}(1,0)=\frac{q}{q^{2}+q+1} z \text { and } f_{z, w}(0,1)=\frac{q}{q^{2}+q+1} w
$$


(where the normalization constant is irrelevant, but useful in view of later representations of these functions). By (2.1), the mappings

$$
(z, w) \mapsto f_{z . w}(m, n)=: C_{m, n}^{q}(z, w) \quad\left(z, w \in \mathbb{N}_{0}\right)
$$

satisfy the recurrence relations

$$
\begin{aligned}
& C_{0, n}^{q} C_{1,0}^{q}=\frac{1}{q^{2}+q+1}\left(\left(q^{2}+q\right) C_{1, n}^{q}+C_{0, n-1}^{q}\right), \\
& C_{m, 0}^{q} C_{1,0}^{q}=\frac{1}{q^{2}+q+1}\left(q^{2} C_{m+1,0}^{q}+(q+1) C_{m-1,1}^{q}\right), \\
& C_{m, n}^{q} C_{1,0}^{q}=\frac{1}{q^{2}+q+1}\left(q^{2} C_{m+1, n}^{q}+q C_{m-1, n+1}^{q}+C_{m, n-1}^{q}\right), \\
& C_{m, 0}^{q} C_{0,1}^{q}=\frac{1}{q^{2}+q+1}\left(\left(q^{2}+q\right) C_{m, 1}^{q}+C_{m-1,0}^{q}\right), \\
& C_{0, n}^{q} C_{0,1}^{q}=\frac{1}{q^{2}+q+1}\left(q^{2} C_{0, n+1}^{q}+(q+1) C_{1, n-1}^{q}\right), \\
& C_{m, n}^{q} C_{0,1}^{q}=\frac{1}{q^{2}+q+1}\left(q^{2} C_{m, n+1}^{q}+q C_{m+1, n-1}^{q}+C_{m-1, n}^{q}\right) \quad(m, n \geq 1) .
\end{aligned}
$$

Induction on $m+n$ shows that for $m, n \in \mathbb{N}_{0}, C_{m, n}^{q}$ is a polynomial in $z, w$ of degree $m+n$ having the form $C_{m, n}^{q}(z, w)=c_{m, n}^{q} z^{m} w^{n}+p_{m, n}^{q}(z, w)$ with $\operatorname{deg} p_{m, n}^{q} \leq m+n-1$ and, for $m, n \geq 1$,

$$
c_{m, 0}^{q}=c_{0, m}^{q}=\frac{1}{q^{m-2}\left(q^{2}+q+1\right)}, \quad c_{m, n}^{q}=\frac{1}{q^{m+n-4}\left(q^{2}+q\right)\left(q^{2}+q+1\right)} .
$$

The $C_{m, n}^{q}$ were constructed in [CM]. To describe this construction, put

$$
S:=\left\{s=\left(s_{1}, s_{2}, s_{3}\right) \in \mathbb{C}^{3}: s_{1} s_{2} s_{3}=1\right\} .
$$

Then the continuous mapping

$$
T: S \rightarrow \mathbb{C}^{2}, \quad s \mapsto(z(s), w(s)):=\left(s_{1}+s_{2}+s_{3}, s_{1}^{-1}+s_{2}^{-1}+s_{3}^{-1}\right)
$$

is onto (notice that for $z, w \in \mathbb{C}$ the polynomial $X^{3}-z X^{2}+w X-1 \in \mathbb{C}[X]$ has zeros $s_{1}, s_{2}, s_{3}$ with $s=\left(s_{1}, s_{2}, s_{3}\right) \in S$ and $z=z(s)$ and $\left.w=w(s)\right)$. Moreover, let $S_{3}$ be the group of all permutations of $\{1,2,3\}$,

$$
K:=K(q):=\frac{1}{(q+1)\left(q^{2}+q+1\right)},
$$

and

$$
c\left(s_{1}, s_{2}, s_{3}\right):=\left(\frac{s_{1}-s_{2} / q}{s_{1}-s_{2}}\right)\left(\frac{s_{1}-s_{3} / q}{s_{1}-s_{3}}\right)\left(\frac{s_{2}-s_{3} / q}{s_{2}-s_{3}}\right) .
$$

The following was shown in [CM, Section 3]. 
PROPOSITION 2.2. Let $q \geq 1$. Then there is a unique family $\left(C_{m, n}^{q}\right)_{m, n}$ of polynomials with $\operatorname{deg} C_{m, n}^{q}=m+n$ which satisfies (2.3) and

$$
C_{1,0}^{q}(z, w)=\frac{q}{q^{2}+q+1} z \quad \text { and } \quad C_{0,1}^{q}(z, w)=\frac{q}{q^{2}+q+1} w .
$$

The $C_{m, n}^{q}$ are given as follows:

(1) If $s \in S$ with $s_{i} \neq s_{j}$ for $i \neq j$, then

$$
C_{m, n}^{q}(z(s), w(s))=\frac{K q^{3}}{q^{m+n}} \sum_{\sigma \in S_{3}} s_{\sigma 1}^{m} s_{\sigma 3}^{-n} c\left(s_{\sigma 1}, s_{\sigma 2}, s_{\sigma 3}\right) .
$$

(2) If $s \in S$ with $s_{2}=s_{3} \neq s_{1}$, then

$$
\begin{aligned}
C_{m, n}^{q}(z(s), w(s))= & K q^{-(m+n)}\left[\left(k_{1,2}^{+}+n k_{1,2}^{-}\right) \frac{s_{1}^{m}}{s_{2}^{n}}\right. \\
& \left.+\left(k_{2,1}^{+}+m k_{2,1}^{-}\right) \frac{s_{2}^{m}}{s_{1}^{n}}+\left(k_{2,2}+(m+n) \tilde{k}_{2,2}\right) s_{2}^{m-n}\right]
\end{aligned}
$$

with

$$
\begin{gathered}
k_{i, j}^{ \pm}:=\frac{(q \pm 1)\left(s_{i} q-s_{j}\right)^{2}}{\left(s_{i}-s_{j}\right)^{2}}, \quad k_{2,2}:=\frac{q(q+1)\left(s_{1}^{2}+s_{2}^{2}\right)-2\left(q^{3}+1\right) s_{1} s_{2}}{\left(s_{1}-s_{2}\right)^{2}}, \\
\text { and } \quad \tilde{k}_{2,2}:=\frac{-(q-1)\left(s_{1} q-s_{2}\right)\left(s_{2} q-s_{1}\right)}{\left(s_{2}-s_{1}\right)^{2}} .
\end{gathered}
$$

(3) If $s \in S$ with $s_{1}=s_{2}=s_{3}$, then

$$
C_{m, n}^{q}(z(s), w(s))=K s_{1}^{m-n} Q(m, n) /\left(2 q^{m+n}\right)
$$

with

$$
\begin{aligned}
Q(m, n):= & (q-1)^{3} m n(m+n)+(q-1)^{2}(q+1)\left(m^{2}+4 m n+n^{2}\right) \\
& +3(q-1)(q+1)^{2}(m+n)+2(q+1)\left(q^{2}+q+1\right) .
\end{aligned}
$$

REMARKS 2.3. (1) For all indices $q$ belonging to triangle buildings, $\left(C_{m, n}^{q}\right)_{m, n \geq 0}$ admit a product formula $C_{m, n}^{q} C_{k, l}^{q}=\sum_{r, s \in \mathbb{N}_{0}} h_{(m, n),(k . l),(r, s)}^{q} C_{r, s}^{q}$ with unique nonnegative linearization coefficients which have the following interpretation in terms of an underlying building $\Gamma$ :

$$
h_{(m, n),(k, l),(r, s)}^{q}=\frac{N_{r, s}}{N_{m, n} N_{k, l}}\left|S_{m, n}(u) \cap S_{l, k}(v)\right|
$$

for any vertices $u, v \in \Gamma$ with $u \in S_{s, r}(v)$; see Section 1 for the notation. Notice that (2.7) is independent of the particular triangle building and depends on $q$ only (see $[\mathrm{CM}]$ ); this means that all buildings of some fixed order $q$ lead to the same hypergroup structure on $\mathbb{N}_{0}^{2}$. 
(2) A comparison of Proposition 2.2 (1) with [Mac2, page 208] shows that for any $q \in \mathbb{R}$ with $q \geq 1$, the $\left(C_{m, n}^{q}\right)_{m, n \geq 0}$ defined in Proposition 2.2 can be written in terms of the Hall-Littlewood polynomials as $C_{m, n}^{q}(z(s), w(s))=P_{(m+n, n, 0)}\left(s_{1}, s_{2}, s_{3} ; 1 / q\right)$. [Mac2, Chapter III.3] therefore shows that the linearization coefficients $h_{(m, n),(k, l),(r, s)}^{q}$ are certain Hall polynomials (in $q$ ) up to certain positive normalization constants; see [Mac2, Chapter II] for an extensive discussion of these polynomials. It was shown by Miller Maley $[\mathrm{M}]$ that the Hall polynomials are in fact polynomials in $(q-1)$ with nonnegative integer coefficients which in particular implies that all $h_{(m, n),(k, l),(r, s)}^{q}$ are nonnegative for all $q \in \mathbb{R}$ with $q \geq 1$. As all preceding results remain correct for all $q \geq 1$, these polynomials define a polynomial hypergroup structure for each $q \in \mathbb{R}$ with $q \geq 1$. Without additional effort we therefore may assume from now on that $q \geq 1$ holds (where sometimes the case $q=1$ has to be excluded).

(3) For $q=1$, the $\left(C_{m, n}^{q}\right)_{m, n \geq 0}$ and the associated hypergroup are related to the building that belongs to a regular tessellation of the euclidian plane by regular triangles. To be more precise, consider the discrete subgroup $L=\left\{m+n e^{2 \pi i / 3}: m, n \in \mathbb{Z}\right\}$ of $(\mathbb{C},+)$ on which the dihedral group $D_{3}$ generated by the reflections on the lines $\mathbb{R} \cdot 1, \mathbb{R} \cdot e^{2 \pi i / 3}$ and $\mathbb{R} \cdot e^{4 \pi i / 3}$ acts as group of automorphisms. The semidirect product $G:=L \times D_{3}$ is a group of type-rotating automorphisms of the canonical graph belonging to $L$ with $H:=D_{3}$ as stabilizer. Therefore, the associated hypergroup according to (1.7) is just $G / / H$.

We next present $\widehat{\mathbb{N}}_{0}^{2}$ and the Plancherel measure for $q \geq 1$.

THEOREM 2.4. The dual space

$$
\widehat{\mathbb{N}}_{0}^{2}:=\left\{\alpha \in C_{b}\left(\mathbb{N}_{0}^{2}\right):\left(\delta_{(m, n)} * \delta_{(a, b)^{-}}\right)(\alpha)=\alpha(m, n) \overline{\alpha(b, a)} \quad \forall m, n, a, b \in \mathbb{N}_{0}\right\}
$$

is given by $\widehat{\mathbb{N}}_{0}^{2}=\left\{f_{z, \bar{z}}: z \in Z_{q}\right\}$, where $Z_{q}$ is the compact closed region in $\mathbb{C}$ bounded by the closed Jordan curve $\gamma_{q}(t):=e^{2 i t}+\left(q+q^{-1}\right) e^{-i t}, t \in[0,2 \pi]$. Moreover, the mapping $I: Z_{q} \rightarrow \widehat{\mathbb{N}}_{0}^{2}, z \mapsto f_{z, \bar{z}}$ is a homeomorphism (where $\widehat{\mathbb{N}}_{0}^{2}$ carries the topology of pointwise convergence). If one identifies $\widehat{\mathbb{N}}_{0}^{2}$ and $Z_{q}$ (which we shall do from now on), then the Plancherel measure $\pi$ on $\widehat{\mathbb{N}}_{0}^{2}$ associated with $\omega$ is the probability measure $d \pi(z)=\left.R_{q}(z) d z\right|_{z_{1}}$, where $d z$ is the usual Lebesgue measure on $\mathbb{C} \simeq \mathbb{R}^{2}$,

$$
\begin{aligned}
& R_{q}(z):=\frac{1}{12 \pi^{2}} \\
& \times \frac{q^{4}(q+1)\left(q^{2}+q+1\right) \sqrt{4\left(z^{3}+\bar{z}^{3}\right)-z^{2} \bar{z}^{2}-18 z \bar{z}+27}}{q^{3}(q+1)^{2}\left(z^{3}+\bar{z}^{3}\right)-q^{4} z^{2} \bar{z}^{2}-q^{2}\left(q^{2}+4 q+1\right)\left(q^{2}+q+1\right) z \bar{z}+q\left(q^{2}+q+1\right)^{3}},
\end{aligned}
$$

and $Z_{1} \subset Z_{q}$ is the compact region bounded by Steiner's hypocycloid.

PROOF. By (2.1) and (2.2), a multiplicative function $f_{z, w}$ on $\mathbb{N}_{0}^{2}$ satisfies

$$
f\left((m, n)^{-}\right)=f((n, m))=\overline{f((m, n))}
$$


for $m, n \in \mathbb{N}_{0}$ if and only if $w=\bar{z}$ holds. It was shown in [MZ] (see also below) that

$$
M:=\left\{z \in \mathbb{C}: f_{z, \bar{z}} \text { bounded on } \mathbb{N}_{0}^{2}\right\}=Z_{q} \supset Z_{1} .
$$

This implies $\widehat{\mathbb{N}}_{0}^{2}=\left\{f_{z, \bar{z}}: z \in Z_{q}\right\}$. As the mapping $I$ is injective by (2.2), surjective by (2.8), and continuous with a compact range, $I$ is a homeomorphism. Finally, the Plancherel measure $\pi$ was determined in [CM, Proposition 5.2] (notice that the proof there carries over to the case $q \geq 1$ ). Finally, $\pi$ is a probability measure due to the normalization $\omega((0,0))=1$.

We here notice that the identity character $1 \in \widehat{\mathbb{N}}_{0}^{2}$ is given by $\mathbf{1}=f_{z_{q}, z_{q}}$ with $z_{q}:=\left(q^{2}+q+1\right) / q$, which belongs to the boundary of $Z_{q}$.

As the proof of (2.8) in [MZ] is quite long, we give a compact proof. According to [CMS, page 230], the subset

$$
S_{\text {sym }}:=\left\{s=\left(s_{1}, s_{2}, s_{3}\right) \in \mathbb{C}^{3}: s_{1} s_{2} s_{3}=1, \bar{s}_{1}+\bar{s}_{2}+\bar{s}_{3}=s_{1}^{-1}+s_{2}^{-1}+s_{3}^{-1}\right\}
$$

of $S$ can be described as follows:

LEMMA 2.5. $S_{\mathrm{sym}}=H_{1} \cup H_{2}$ with

$$
\begin{aligned}
& H_{1}:=\left\{\left(e^{i \vartheta_{1}}, e^{i \vartheta_{2}}, e^{-i\left(\vartheta_{1}+\vartheta_{2}\right)}\right): \vartheta_{1}, \vartheta_{2} \in[0,2 \pi]\right\} \\
& H_{2}:=\left\{\sigma\left(e^{i 2 \vartheta}, r e^{-i \vartheta}, r^{-1} e^{-i \vartheta}\right): \sigma \in S_{3}, r>0, \vartheta \in[0,2 \pi]\right\} .
\end{aligned}
$$

Moreover, we have the following description of the set $M$.

LemMA 2.6. If $S_{q}:=\left\{s \in S_{\mathrm{sym}}: q^{-1} \leq\left|s_{i}\right| \leq q\right.$ for $\left.i=1,2,3\right\}$, then $M=$ $\left\{s_{1}+s_{2}+s_{3}: s \in S_{q}\right\}$.

PRoOf. Let $s \in S_{q}$. If $s_{i} \neq s_{j}$ for $i \neq j$, then by Proposition $2.2(1), z(s):=$ $s_{1}+s_{2}+s_{3}$ satisfies

$$
\sup _{m, n \in \mathbb{N}_{0}}\left|C_{m, n}^{q}(z(s), \overline{z(s)})\right| \leq \sup _{m, n \in \mathbb{N}_{0}} \frac{K q^{3}}{q^{m+n}} \sum_{\sigma \in S_{3}}\left|c\left(s_{\sigma 1}, s_{\sigma 2}, s_{\sigma 3}\right)\right| q^{m+n}<\infty
$$

and hence $z(s) \in M$. The other cases follow similarly. To check the reverse inclusion, consider $s \in S_{\text {sym }} \backslash S_{q}$. By Lemma 2.5 and symmetry, we may assume that $\left|s_{1}\right|=1$, $\left|s_{2}\right|>q$ and $\left|s_{3}\right|<1 / q$. Then, by Proposition 2.2 (1),

$$
\left|C_{m, 0}^{q}(z(s), \overline{z(s)})\right|=\frac{K q^{3}}{q^{m}}\left|\sum_{\sigma \in S_{3}} s_{\sigma 1}^{m} c\left(s_{\sigma 1}, s_{\sigma 2}, s_{\sigma 3}\right)\right| \rightarrow \infty
$$

as $m \rightarrow \infty$ since $c\left(s_{2}, s_{1}, s_{3}\right)+c\left(s_{2}, s_{3}, s_{1}\right) \neq 0$. Thus $z(s) \notin M$. 
To complete the proof of (2.8), we claim that for $r \geq 1$ the set

$$
Z_{r}:=\left\{s_{1}+s_{2}+s_{3}:\left(s_{1}, s_{2}, s_{3}\right) \in S_{r}\right\}
$$

is the compact closed region in $\mathbb{C}$ bounded by the closed Jordan curve

$$
\gamma_{r}(t):=e^{2 i t}+\left(r+r^{-1}\right) e^{-i t} \quad(t \in[0,2 \pi]) .
$$

For this, use Lemma 2.5 and write $Z_{r}=Z_{r}^{1} \cup Z_{r}^{2}$ with

$$
Z_{r}^{i}=\left\{s_{1}+s_{2}+s_{3}:\left(s_{1}, s_{2}, s_{3}\right) \in S_{r} \cap H_{i}\right\}
$$

where, by the definition of $\left.\left.H_{2}, Z_{r}^{2}=\left\{\gamma_{s}(t): s \in\right] 1, r\right], t \in[0,2 \pi]\right\}$. It can be checked (see [CM, Proposition 4.5]) that $Z_{r}^{1}$ is the compact closed region in $\mathbb{C}$ bounded by the hypocycloid $\gamma_{1}$. As for $r>s \geq 1, \gamma_{s}$ is a Jordan curve contained in the interior of $\gamma_{r}$, (2.8) follows.

REMARK 2.7. (1) Let $q>1$ and $p>2$. Then a character $f_{z, \bar{z}} \in \widehat{\mathbb{N}}_{0}^{2}$ belongs to $L^{p}\left(\mathbb{N}_{0}^{2}, \omega\right)$ if and only if $z \in Z_{q^{1-2 / p}}$ holds. This follows readily from Proposition 2.1 and the methods in the proof of Lemma 2.6; this was already observed in [MZ] for prime powers $q$.

(2) Let $q>1$. Then the character $f_{3,3}$ belongs to $L^{p}\left(\mathbb{N}_{0}^{2}, \omega\right)$ for all $p>2$ and is positive and in the support of the Plancherel measure. Therefore, by [BH, Theorem 2.5.6], $\mathbb{N}_{0}^{2}$ has the Kunze-Stein property for any $p>2$. This fact was derived in a different way in [MZ].

REMARK 2.8. (1) (2.1) and induction yield, for $m, n, k, l \in \mathbb{N}_{0}$,

$$
\operatorname{supp}\left(\delta_{(m, n)} * \delta_{(k, l)}\right) \subset\left\{(u, v) \in \mathbb{N}_{0}^{2}: u-v \equiv m-n+k-l \bmod 3\right\} .
$$

This means that $L_{0}:=\left\{(m, n) \in \mathbb{N}_{0}: m \equiv n \bmod 3\right\}$ is a supernormal subhypergroup of $\mathbb{N}_{0}^{2}$ in the language of hypergroups; see [BH].

(2) (2.3) and induction imply that for all $m, n \in \mathbb{N}_{0}$ and $z \in \mathbb{C}$,

$$
C_{m, n}^{q}\left(e^{2 \pi i / 3} z, e^{-2 \pi i / 3} \bar{z}\right)=e^{2 \pi(m-n) i / 3} C_{m, n}^{q}(z, \bar{z}) ;
$$

see [CM, Section 3]. This equation is obviously connected with the supernormality of $L_{0}$.

Finally, we introduce the Fourier transform of measures $\mu \in M_{b}\left(\mathbb{N}_{0}^{2}\right)$ by

$$
\hat{\mu}(z):=\sum_{m, n \in \mathbb{N}_{0}} \overline{C_{m, n}^{q}(z, \bar{z})} \mu(\{(m, n)\}) \quad\left(z \in Z_{q} \simeq \widehat{\mathbb{N}}_{0}^{2}\right) .
$$

As $\left|C_{m, n}^{q}(z, \bar{z})\right| \leq 1$ for $z \in Z_{q}$, the function $\hat{\mu}$ is continuous on $Z_{q}$ with $\|\hat{\mu}\|_{\infty} \leq\|\mu\|$. For further basic properties of the Fourier transform on commutative hypergroups we refer to $[\mathrm{BH}]$. 


\section{Expansions into generalized Tchebychev polynomials}

Here we expand the polynomials $C_{m, n}^{q}$ in terms of certain Tchebychev polynomials $\left(T_{m, n}\right)_{m, n \geq 0}$ and $\left(U_{m, n}\right)_{m, n \geq 0}$ of the first and second kind respectively which are orthogonal polynomials with respect to certain canonical measures on the region $Z_{1}$ bounded by Steiner's hypocycloid. We define these polynomials according to [Kw]. Each $z \in Z_{1}$ can be written as $z=z(s, t):=e^{i s}+e^{-i t}+e^{i(-s+t)}(s, t \in[0,2 \pi])$. For $m, n \in \mathbb{N}_{0}$, put

$$
\begin{aligned}
e_{m, n}^{ \pm}(s, t):= & e^{i(m s+n t)} \pm e^{i((m+n) s-n t)}+e^{i(-(m+n) s+m t)} \\
& \pm e^{i(-n s-m t)}+e^{i(n s-(m+n) t)} \pm e^{i(-m s+(m+n) t)}, \quad(m, n \geq 1), \\
e_{m, 0}^{+}(s, t):= & e^{i m s}+e^{-i m t}+e^{i(-m s+m t)}, \quad e_{0, m}^{+}(s, t)=e_{m, 0}^{+}(t, s), \quad(m \geq 1) \\
e_{0.0}^{+}(s, t):= & \text { and } \quad e_{0, m}^{-}=e_{m, 0}^{-}=0, \quad(m \geq 0) .
\end{aligned}
$$

In $[\mathrm{Kw}]$, Koornwinder derived the following result:

PROPOSITION 3.1. For all $m, n \in \mathbb{N}_{0}$ there exist unique polynomials $T_{m, n}, U_{m, n} \in$ $\mathbb{C}[z, \bar{z}]$ such that for all $z=z(s, t)$ in the interior of $Z_{1}$

$$
T_{m, n}(z, \bar{z})=e_{m, n}^{+}(s, t) \quad \text { and } \quad U_{m, n}(z, \bar{z})=\frac{e_{m+1, n+1}^{-}(s, t)}{e_{1,1}^{-}(s, t)}
$$

holds with $e_{1,1}^{-}(s, t) \neq 0$. The polynomials have the form $T_{m, n}(z, \bar{z})=z^{m} \bar{z}^{n}+\pi_{m, n}$ and $U_{m, n}(z, \bar{z})=z^{m} \bar{z}^{n}+\tilde{\pi}_{m, n}$ with $\pi_{m, n}, \tilde{\pi}_{m, n} \in \mathbb{C}[z, \bar{z}]$ of degree at most $m+n-1$. The $\left(T_{m, n}(z, \bar{z})\right)_{m, n \geq 0}$ and $\left(U_{m, n}(z, \bar{z})\right)_{m, n \geq 0}$ are orthogonal polynomials with respect to the measures $\left.R_{1}(z) d z\right|_{z_{1}}$ and $\left.R_{1}(z)^{-1} d z\right|_{Z_{1}}$ respectively on $Z_{1}$ with $R_{1}$ as in Theorem 2.4 .

REMARK 3.2. (1) By Weyl's character formula for $S U$ (3) (see, for instance, [Si, Theorem IX.9.1]), we may regard $\left\{U_{m, n}: m, n \geq 0\right\}$ as the set of all characters of $S U(3)$, where the character associated with $U_{m, n}$ at some element of $S U(3)$ with eigenvalues $e^{i s}, e^{-i t}, e^{i(t-s)}$ for $s, t \in[0,2 \pi]$ is given by $U_{m, n}(z(s, t), \overline{z(s, t)})$. In particular, the $U_{m, n}$ admit a product formula with nonnegative linearization coefficients which corresponds to the decomposition of tensor products of the associated irreducible unitary representations.

(2) By Proposition $2.2(1)$, the polynomials $C_{m, n}^{1}$ and $T_{m, n}$ are related by $C_{m, n}^{1}=$ $T_{m, n} / 6, C_{m, 0}^{1}=T_{m, 0} / 3, C_{0, m}^{1}=T_{0, m} / 3, C_{0,0}^{1}=T_{0,0}(m, n \geq 1)$.

We now turn to the expansion of $C_{m, n}^{q}$ in terms of $T_{m, n}$ for $q \geq 1$.

PROPOSITION 3.3. The connection coefficients $h_{m, n, r, s}=h_{m, n, r, s}(q)$ of

$$
C_{m, n}^{q}=\sum_{r, s \in \mathbb{N}_{0}} h_{m, n, r, s} T_{r, s} \quad\left(m, n \in \mathbb{N}_{0}\right)
$$


satisfy $h_{m, n, r, s} \geq 0$.

A similar positivity result for quite general one-dimensional orthogonal polynomials was given by [LR]. Proposition 3.3 follows from the following trivial observation and Lemma 3.5 below.

REMARK 3.4. Consider the polynomial $p\left(s_{1}, s_{2}, s_{3}\right):=\sum_{\sigma \in S_{3}} s_{\sigma 1}^{k} s_{\sigma 2}^{l} s_{\sigma 3}^{m}$ with $0 \leq m \leq l \leq k$. Then, for $s, t \in \mathbb{R}$ and $\left(s_{1}, s_{2}, s_{3}\right):=\left(e^{i s}, e^{-i t}, e^{i(-s+l)}\right)$,

$$
p\left(s_{1}, s_{2}, s_{3}\right)=\sum_{\sigma \in S_{3}} \frac{s_{\sigma 1}^{k} s_{\sigma 2}^{l} s_{\sigma 3}^{m}}{\left(s_{\sigma 1} s_{\sigma 2} s_{\sigma 3}\right)^{l}}=\sum_{\sigma \in S_{3}} \frac{s_{\sigma 1}^{k-l}}{s_{\sigma 3}^{l-m}}=e_{k-1, t-m}^{+}(s, t) .
$$

Therefore, for each symmetric polynomial $p \in \mathbb{C}\left[s_{1}, s_{2}, s_{3}\right]$ with nonnegative coefficients there exist $n_{0} \in \mathbb{N}$ and coefficients $h_{k, l} \geq 0\left(0 \leq k, l \leq n_{0}\right)$ with

$$
p\left(e^{i s}, e^{-i t}, e^{i(-s+t)}\right)=\sum_{k, l=0}^{n_{0}} h_{k, l} T_{k, l}(z(s, t), \overline{z(s, t)}), \quad \forall s, t \in \mathbb{R} .
$$

Proposition 3.3 is now a consequence of the following lemma, which is Proposition 3.5 in [CM]. Note that the proof there needs correcting: the numerators $s_{\alpha 1} x$ and $s_{\sigma 1} x$ in the expression for $F(x, y)$ in line 3 of the proof there should be 1 . The lemma also follows from [CMS, Proposition 3.1].

LEMMA 3.5. Let $q \geq 1$. For $m, n \in \mathbb{N}_{0}$, the rational function

$$
S=\left\{s \in \mathbb{C}^{3}: s_{1} s_{2} s_{3}=1\right\} \rightarrow \mathbb{C}, \quad s \mapsto C_{m, n}^{q}\left(s_{1}+s_{2}+s_{3}, s_{1}^{-1}+s_{2}^{-1}+s_{3}^{-1}\right)
$$

is a symmetric polynomial in $s_{1}, s_{2}, s_{3}$ with nonnegative coefficients.

REMARK 3.6. (1) For $m, n \in \mathbb{N}_{0}$ and $s \in S$ with $s_{i} \neq s_{j}$ for $i \neq j$,

$$
\tilde{C}_{m, n}^{q}(s):=q^{3} \sum_{\sigma \in S_{3}} s_{\sigma 1}^{m+n} s_{\sigma 2}^{n} \cdot c\left(s_{\sigma 1}, s_{\sigma 2}, s_{\sigma 3}\right)
$$

is a polynomial in $q$ of the form $\tilde{C}_{m, n}^{q}(s)=\sum_{j=0}^{3} x_{j}(q-1)^{j}$, where the $x_{l}=x_{l}^{m . n}(s)$ are symmetric rational functions in $s_{1}, s_{2}, s_{3}$. It can be checked (see [CM]) that these functions are polynomials in $s_{1}, s_{2}, s_{3}$ with nonnegative coefficients (which leads to a further proof of Lemma 3.5). In particular, it is easy to see that $x_{0}^{m, n}=\sum_{\sigma \in S_{3}} s_{\sigma 1}^{m+n} s_{\sigma 2}^{n}$ and $x_{3}^{m, n}=\sum_{i=0}^{m} \sum_{j=0}^{n} \sum_{k=0}^{i+j} s_{1}^{m-i+j} s_{2}^{n+i-k} s_{3}^{n+k-j}$. In particular,

$$
x_{0}^{m, n}\left(e^{i s}, e^{-i t}, e^{-i(s-t)}\right)=T_{m, n}(z(s, t), \overline{z(s, t)})
$$

implies that $h_{m, n, m, n} \geq K / q^{m+n}$. 
(2) Each $x \in \mathbb{B}$ with $x \geq 3$ can be written as $x=x(r)=1+r+r^{-1}$ with $r \in \mathbb{R}$, $r \geq 1$; in this case,

$$
T_{m, n}(x(r), x(r))=r^{m}+\frac{1}{r^{m}}+r^{n}+\frac{1}{r^{n}}+r^{m+n}+\frac{1}{r^{m+n}}>0 .
$$

Proposition 3.3 hence yields $C_{m, n}^{q}(x, x)>0$ for $q \geq 1$ and $x \in \mathbb{R}$ with $x \geq 3$. Moreover, (3.3) implies that for $\eta \in[0, \ln q]$ and $m, n \in \mathbb{N}_{0}$,

$$
T_{m, n}\left(x\left(e^{\eta} / q\right), x\left(e^{\eta} / q\right)\right) \geq e^{-(m+n) \eta} T_{m, n}(x(q), x(q)) .
$$

As $C_{m, n}^{q}(x(q), x(q))=1$ for $m, n \in \mathbb{N}_{0}$, we obtain for $m, n \in \mathbb{N}_{0}$,

$$
C_{m, n}^{q}\left(x\left(e^{\eta} / q\right), x\left(e^{\eta} / q\right)\right) \geq e^{-(m+n) \eta} \quad(\eta \in[0, \ln q]) .
$$

(3) The definition of the $T_{m, n}$ shows that $\left|T_{m, n}(z, \bar{z})\right| \leq T_{m, n}(3, \overline{3})$ for all $m, n \in \mathbb{N}_{0}$, $z \in Z_{1}$. It follows from Proposition 3.3 that for all $q \geq 1$,

$$
\left|C_{m, n}^{q}(z, \bar{z})\right| \leq C_{m, n}^{q}(3, \overline{3}) \text { for all } m, n \in \mathbb{N}_{0}, z \in Z_{1} .
$$

(4) Computing $T_{m, n}(3,3)$ and $C_{m, n}^{q}(3,3)$ (see Proposition $2.2(3)$ ), we see that for each $q \geq 1$ there is a polynomial $Q^{q} \in \mathbb{R}[x, y]$ with

$$
\sum_{k, l} h_{m, n, k, l} \leq \frac{Q^{q}(m, n)}{q^{m+n}} \text { for } m, n \in \mathbb{N}_{0} .
$$

In Section 7 we need the following technical results about the $h_{m, n, k, l}$ :

LEMMA 3.7. Let $q>1$ and $m, n \in \mathbb{N}_{0}$ with $m \geq n$.

(1) If $(m, n) \in L_{0}$, then $h_{m, n, 0,0}>0$. Moreover, if $(m, n) \in L_{0} \backslash\{(0,0)\}$, then $h_{m, n, 1,1}>0$.

(2) If $m-n \equiv 1 \bmod 3$, then $h_{m, n, 1,0}>0$. Moreover, if additionally $(m, n) \neq$ $(1,0)$, then $h_{m, n, 0,2}>0$.

PROOF. By Remark 3.6 (1) it suffices to check that the corresponding coefficients in $x_{3}^{m, n}$ are positive, and this follows from Remark 3.6 (1) and Remark 3.4.

We next turn to expansions in terms of the $U_{m, n}$.

PROPOSITION 3.8. For $q \geq 1$, the polynomials $C_{m, n}^{q}$ satisfy

$$
\begin{gathered}
C_{0.0}^{q}=U_{0,0}, \quad C_{1,0}^{q}=\frac{q}{q^{2}+q+1} U_{1,0}, \quad C_{1,1}^{q}=\frac{K}{q^{2}}\left[q^{3} U_{1.1}-q(q+1)\right], \\
C_{2,1}^{q}=\frac{K}{q^{3}}\left[q^{3} U_{2,1}-q^{2}\left(U_{1,0}+U_{0,2}\right)\right], \quad C_{2,0}^{q}=\frac{K}{q^{2}}\left[q^{2}(q+1) U_{2,0}-q(q+1) U_{0,1}\right], \\
C_{2.2}^{q}=\frac{K}{q^{4}}\left[q^{3} U_{2,2}-q^{2}\left(U_{3,0}+U_{0.3}+U_{1,1}\right)+q U_{1,1}-1\right] .
\end{gathered}
$$


Moreover, for $m, n \geq 3$,

$$
\begin{aligned}
C_{m, 0}^{q}= & \frac{K}{q^{m}}\left[q^{2}(q+1) U_{m, 0}-q(q+1) U_{m-2,1}+(q+1) U_{m-3,0}\right], \\
C_{m, 1}^{q}= & \frac{K}{q^{m+1}}\left[q^{3} U_{m, 1}-q^{2}\left(U_{m-2,2}+U_{m-1,0}\right)+q U_{m-3,1}\right], \\
C_{m, 2}^{q}= & \frac{K}{q^{m+2}}\left[q^{3} U_{m, 2}-q^{2}\left(U_{m+1,0}+U_{m-2,3}\right)\right. \\
& \left.-q(q-1) U_{m-1,1}+q U_{m-3,2}-U_{m-2,0}\right], \\
C_{m, n}^{q}= & \frac{K}{q^{m+n}}\left[q^{3} U_{m, n}-q(q-1) U_{m-1, n-1}-U_{m-2, n-2}\right. \\
& \left.-q^{2}\left(U_{m-2, n+1}+U_{m+1, n-2}\right)+q\left(U_{m-3, n}+U_{m, n-3}\right)\right] .
\end{aligned}
$$

Proof. The cases $m, n \leq 2$ can be checked directly. For general $m, n \in \mathbb{N}_{0}$, consider $s, t \in[0, \pi]$ such that $\left(s_{1}, s_{2}, s_{3}\right):=\left(e^{i s}, e^{-i t}, e^{-i(s-t)}\right)$ satisfies $s_{i} \neq s_{j}$ for $i \neq j$. The function $\tilde{C}_{m, n}^{q}$ defined in (3.1) is a polynomial in $q$ with

$$
\tilde{C}_{m, n}^{q}\left(s_{1}, s_{2}, s_{3}\right)=a_{0}+x_{1} q+a_{2} q^{2}+a_{3} q^{3},
$$

where the coefficients $a_{l}=a_{l}^{m, n}(s, t)$ are given as follows. If $m, n \geq 3$, then

$$
a_{0}^{m, n}=-\frac{\sum_{\sigma \in S_{3}} \operatorname{sgn}(\sigma) s_{\sigma 1}^{m+n} s_{\sigma 2}^{n+1} s_{\sigma 3}^{2}}{\left(s_{2}-s_{1}\right)\left(s_{3}-s_{1}\right)\left(s_{3}-s_{2}\right)}=-U_{m-2, n-2}(z(s, t), \overline{z(s, t)}) .
$$

We omit the arguments of $U_{m, n}$. A similar computation shows that $a_{0}^{m, 2}=U_{m-2,0}$, $a_{0}^{m .1}=0$, and $a_{0}^{m, 0}=U_{m-3,0}$ for $m \geq 3$. Moreover, for $m, n \geq 3$,

$$
\begin{aligned}
a_{1}^{m, n} & =\frac{\sum_{\sigma \in S_{3}} \operatorname{sgn}(\sigma) \cdot s_{\sigma 1}^{m+n} s_{\sigma 2}^{n}\left(s_{\sigma 1} s_{\sigma 3}^{2}+s_{\sigma 1} s_{\sigma 2} s_{\sigma 3}+s_{\sigma 2}^{2} s_{\sigma 3}\right)}{\left(s_{2}-s_{1}\right)\left(s_{3}-s_{1}\right)\left(s_{3}-s_{2}\right)} \\
& =\frac{e_{m-2, n+1}^{-}(s, t)+e_{m+1, n-2}^{-}(s, t)+e_{m, n}^{-}(s, t)}{e_{1,1}^{-}(s, t)} \\
& =U_{m-3, n}+U_{m, n-3}+U_{m-1, n-1} .
\end{aligned}
$$

and, similarly, $a_{1}^{m, 2}=U_{m-1,1}+U_{m-3,2}$ as well as $a_{1}^{m, 1}=U_{m-3,1}$ and $a_{1}^{m, 0}=-U_{m-2,1}+$ $U_{m-3,0}$. Furthermore, for $m, n \geq 2$,

$$
\begin{aligned}
a_{2}^{m, n} & =-\frac{\sum_{\sigma \in S_{3}} \operatorname{sgn}(\sigma) \cdot s_{\sigma 1}^{m+n} s_{\sigma 2}^{n}\left(s_{\sigma 1}^{2} s_{\sigma 3}++s_{\sigma 1} s_{\sigma 2} s_{\sigma 3}+s_{\sigma 1} s_{\sigma 2}^{2}\right)}{\left(s_{2}-s_{1}\right)\left(s_{3}-s_{1}\right)\left(s_{3}-s_{2}\right)} \\
& =-\frac{e_{m+2, n-1}^{-}(s, t)+e_{m-1, n+2}^{-}(s, t)+e_{m, n}^{-}(s, t)}{e_{1,1}^{-}(s, t)} \\
& =-\left(U_{m+1, n-2}+U_{m-2, n+1}+U_{m-1, n-1}\right),
\end{aligned}
$$


and, similarly, $a_{2}^{m, 1}=-U_{m-2,2}-U_{m-1,0}$ and $a_{2}^{m, 0}=U_{m, 0}-U_{m-2,1}$. Finally,

$$
a_{3}^{m, n}=\frac{\sum_{\sigma \in S_{3}} \operatorname{sgn}(\sigma) \cdot s_{\sigma 1}^{m+n+2} s_{\sigma 2}^{n+1}}{\left(s_{2}-s_{1}\right)\left(s_{3}-s_{1}\right)\left(s_{3}-s_{2}\right)}=\frac{e_{m+1, n+1}^{-}(s, t)}{e_{1,1}^{-}(s, t)}=U_{m, n}
$$

for all $m, n$. Putting all results together, we obtain the proposition.

\section{Moment functions}

Moment functions on commutative hypergroups are $\mathbb{R}$-valued functions with additive properties similar to the multiplicativity of characters; they generalize the functions $x \mapsto x^{n}$ on $(\mathbb{R},+)$. As shown in [BH, V1, V2, Z1, Z2], they provide a useful tool in probability theory. In many cases, they can be constructed as directional derivatives in the dual space at the identity character 1 . We thus need a suitable parameterization of some neighborhood of 1 in the dual which will be identified with $Z_{q}$ according Section 2. We restrict our attention to the case $q>1$ ( $q=1$ must be treated separately). In contrast to previous parameterizations we now consider the mapping

$$
\tilde{z}: \mathbb{C}^{2} \rightarrow \mathbb{C}, \quad(\xi, \eta) \mapsto e^{2 i \xi}+e^{-i \xi}\left(e^{i \eta}+e^{-i \eta}\right)
$$

which maps $\left\{(\xi, \eta) \in \mathbb{C}^{2}: \xi, \operatorname{Re} \eta \in[0,2 \pi],|\operatorname{Im} \eta| \in[-\ln q, \ln q]\right\}$ onto a neighbourhood of 1 according to Section 2, where 1 corresponds to $z_{q}=1+q+1 / q=$ $z(0, i \ln (q))$. We are now ready to define moment functions.

DEFINITION 4.1. For $r, s, m, n \in \mathbb{N}_{0}$ and $\xi, \eta \in \mathbb{C}$, let

$$
\varphi_{r, s, \xi, \eta}(m, n):=\left.i^{r+s} \frac{\partial^{r+s}}{\partial x^{r} \partial y^{s}} C_{m, n}(\tilde{z}(x, y), \overline{\tilde{z}(x, y)})\right|_{(x, y)=(\xi, \eta)} .
$$

The moment function $m_{r, s}$ of index $(r, s) \in \mathbb{N}_{0}^{2}$ is now defined by

$$
m_{r, s}(m, n):=\varphi_{r, s, 0, i \ln q}(m, n) .
$$

EXAMPLE 4.2. A short calculation using the explicit form of $C_{1,0}$ and $C_{0,1}$ shows that $m_{0,1}$ and $m_{1,0}$ are given by

$$
\begin{aligned}
m_{1,0}(m, n)= & m-n+\frac{3 q}{q^{2}-1}\left(\frac{1}{q^{m}}-\frac{1}{q^{n}}\right) \\
& +\frac{3 q^{2}}{\left(q^{2}-1\right)\left(q^{2}+q+1\right)}\left(\frac{1}{q^{m+2 n}}-\frac{1}{q^{2 m+n}}\right) \\
m_{0,1}(m, n)= & m+n+\frac{q}{q^{2}-1}\left(\frac{1}{q^{m}}+\frac{1}{q^{n}}\right)-\frac{2 q\left(q^{2}+2 q+1\right)}{\left(q^{2}-1\right)\left(q^{2}+q+1\right)} \\
& +\frac{q^{2}}{\left(q^{2}-1\right)\left(q^{2}+q+1\right)}\left(\frac{1}{q^{m+2 n}}+\frac{1}{q^{2 m+n}}\right) .
\end{aligned}
$$


The expansion of the $C_{m, n}$ in terms of the $T_{m, n}$ in Section 3 leads to the following representation of the moment functions.

LEMMA 4.3. For all $m, n \in \mathbb{N}_{0}$ there exist real numbers $d_{m, n, k, l} \geq 0$ with

$$
\sum_{k, l \in \mathbb{Z},|k|+|l| \leq 2(m+n)} d_{m, n, k, l}=1
$$

such that for all $r, s \in \mathbb{N}_{0}$,

$$
m_{r, s}(m, n)=\sum_{k, l \in \mathbb{Z},|k|+|l| \leq 2(m+n)} d_{m, n, k, l} k^{r} l^{s}
$$

PROOF. Fix $m, n \in \mathbb{N}_{0}$. Proposition 3.3 and the explicit representation of the Tchebychev polynomials of the first kind in Section 3 imply that there exist constants $c_{m, n, k, l} \geq 0$ such that for all $\xi, \eta \in \mathbb{C}$

$$
C_{m, n}(z(\xi, \eta), \overline{z(\xi, \eta)})=\sum_{k, l \in \mathbb{Z},|k|+|l| \leq 2(m+n)} c_{m, n, k, l} e^{-i k \xi} e^{-i l \eta}
$$

As $C_{m, n}\left(z_{q}, \bar{z}_{q}\right)=1$, the constants $d_{m, n, k, l}:=c_{m, n, k, l} q^{l} \geq 0$ satisfy $(*)$ and

$$
C_{m, n}(z(\xi, \eta), \overline{z(\xi, \eta)})=\sum_{k . l \in \mathbb{Z},|k|+|l| \leq 2(m+n)} d_{m . n . k . l} e^{-i k \xi} e^{-i l(\eta-i \ln q)} .
$$

The lemma now follows by differentiation.

We next collect some properties of the moment functions. We in particular obtain that the pairs $\left(m_{0,1}, m_{0,2}\right)$ and $\left(m_{1,0}, m_{2,0}\right)$ form pairs of moment functions in the sense of Zeuner [Z2]; see [BH, Section 7.2].

PROPOSITION 4.4. The $m_{r . s}$ have the following properties for $r, s \in \mathbb{N}_{0}$ :

(1) For all $m, n, k, l \in \mathbb{N}_{0}$,

$$
\left(\delta_{(m, n)} *\left(\delta_{(k, l)}\right)\left(m_{r, 0}\right)=\sum_{j=0}^{r}\left(\begin{array}{l}
r \\
j
\end{array}\right) m_{j, 0}(m, n) m_{r-j, 0}(k, l) .\right.
$$

The same binomial formula holds for the moment functions $m_{0, r}$.

(2) $m_{1,0}^{r} \leq m_{r, 0}$ and $m_{0,1}^{r} \leq m_{0, r}$.

(3) If $\xi \in \mathbb{R}$ and $\eta \in \mathbb{C}$ with $\operatorname{Im}(\eta) \in[0, \ln q]$, then $\varphi_{r, s, \xi, \eta}(m, n) \leq(2(m+n))^{r+s}$ and $m_{r, s}(m, n) \leq(2(m+n))^{r+s}$.

(4) For each $r \in \mathbb{N}$, there is a constant $C=C(q, r)$ such that for all $m, n \in \mathbb{N}_{0}$, $m_{0, r}(m, n) \geq C(m+n)^{r}$. 
(5) For $m, n, r \in \mathbb{N}_{0}$, the function $\psi^{r, m, n}(\eta):=\varphi_{0, r, 0, i \eta}(m, n)$ is nonnegative and increasing on $[0, \ln q]$ and satisfies for $0 \leq \eta_{1} \leq \eta_{2}<\ln q$

$$
\frac{m_{0, r}(m, n)-\psi^{r, m, n}\left(\eta_{1}\right)}{\ln q-\eta_{1}} \leq \frac{m_{0, r}(m, n)-\psi^{r, m, n}\left(\eta_{2}\right)}{\ln q-\eta_{2}} .
$$

PROOF. (1) This follows immediately from the multiplicativity of the hypergroup characters and the Leibniz rule for derivatives.

(2) This follows from Lemma 4.3 and Jensen's inequality.

(3) This follows from (4.1) in the proof of Lemma 4.3.

(4) This follows from Part (2) together with the explicit representation of $m_{0,1}$ in Example 4.2.

(5) Write $C_{m, n}^{q}$ as a nonnegative linear combination of the $T_{k, l}$ as in Proposition 3.3. Differentiation and the definition of the $T_{k, l}$ then show that $\psi$ is a nonnegative linear combination of nonnegative, increasing and convex functions. As $\psi(\ln q)=m_{0, r}(m, n)$, the lemma is clear.

In the next section we shall use the moment functions above in order to introduce a concept of modified moments of probability measures on $\mathbb{N}_{0}^{2}$. As a preparation we need some further technical results. The following result is a consequence of Parts (3) and (4) of Proposition 4.4.

COROLlaRY 4.5. Let $\mu \in M^{1}\left(\mathbb{N}_{0}^{2}\right)$. Then for any $s \geq 1, \int_{\mathbb{N}_{0}^{2}} m_{0, s} d \mu<\infty$ holds if and only if $\int_{N_{0}^{2}}(m+n)^{s} d \mu(m, n)<\infty$ holds.

Proposition 4.6. Let $\mu \in M^{1}\left(\mathbb{N}_{0}^{2}\right)$ and $N \in \mathbb{N}$. Then

$$
\int_{N_{0}^{2}}(m+n)^{N} d \mu(m, n)<\infty
$$

holds if and only if the function $(\xi, \eta) \mapsto \hat{\mu}(z(\xi, \eta))$ is $N$-times continuously partially differentiable for $(\xi, \eta) \in \mathbb{R} \times\{z \in \mathbb{C}: \operatorname{Im}(z) \in[0, \ln q]\}$. Moreover, if one of these conditions holds, then

$$
\frac{\partial^{r+s}}{\partial \xi^{r} \partial \eta^{s}} \hat{\mu}(z(\xi, \eta))=(-i)^{r+s} \int_{\mathbb{N}_{0}^{2}} \varphi_{r, s, \xi, \eta} d \mu .
$$

Proof. If (4.2) holds, then Proposition 4.4 (3), the dominated convergence theorem, and induction on $r$ and $s$ yield that the mapping above is $N$-times continuously partially differentiable and that (4.3) holds. Assume now that the mapping above is 
$N$-times continuously partially differentiable. Using induction, we may assume, by Corollary 4.5 , that for $0 \leq s \leq N-1$,

$$
\int_{\mathbb{N}_{0}^{2}} m_{0, s}(m, n) d \mu(m, n)<\infty
$$

with

$$
\frac{d^{s}}{d \eta^{s}} \hat{\mu}(z(0, i \eta))=\int_{\mathbb{N}_{0}^{2}} \varphi_{0, s, 0, \eta} d \mu
$$

for $\eta \in[0, \ln q]$. By Proposition 4.4 (5) we have

$$
\frac{m_{0, N-1}(m, n)-\psi^{N-1, m, n}(\eta)}{\ln q-\eta} \uparrow m_{0, N}(m, n) \text { for } \eta \uparrow \ln q,
$$

where $m, n \in \mathbb{N}_{0}$. Hence, by monotone convergence,

$$
\int_{\mathbb{N}_{0}^{2}} m_{0, N}(m, n) d \mu(m, n)=\frac{d}{d \eta}\left(\left.\frac{d^{s}}{d \eta^{s}} \hat{\mu}(z(0, i \eta))\right|_{\eta=\ln q},\right.
$$

where the right-hand side is finite. This completes the proof.

\section{Strong laws of large numbers}

Assume again that $q>1$ holds. Based on the moment functions we first introduce modified moments of $\mathbb{N}_{0}^{2}$-valued random variables. We then use these modified moments to derive some strong laws of large numbers. For practical reasons, it suffices to consider modified moments of order at most two, that is, modified (twodimensional) expectations and variances.

DEFINITION 5.1. Let $X$ be an $\mathbb{N}_{0}^{2}$-valued random variable defined on some probability space $(\Omega, \mathscr{A}, \mathbf{P})$ with distribution $\mu \in M^{1}\left(\mathbb{N}_{0}^{2}\right)$. We say that the first moments of $X$ and $\mu$ exist if $\int_{\mathbb{N}_{0}^{2}}(m+n) d \mu(m, n)<\infty$ holds. Denoting the usual expectation by $\mathbb{E}$, we then put

$$
\mathbb{E}_{1}(X):=\mathbb{E}_{1}(\mu):=\mathbb{E}\left(m_{1.0}(X)\right)=\int_{\mathbb{N}_{0}^{2}} m_{1,0} d \mu
$$

and $\mathbb{E}_{2}(X):=\mathbb{E}_{2}(\mu):=\mathbb{E}\left(m_{0,1}(X)\right)$. Moreover, if $\int_{\mathbb{N}_{0}^{2}}(m+n)^{2} d \mu(m, n)<\infty$, then we say that the second moments exist and put

$$
\vee_{1}(X):=\mathbb{V}_{1}(\mu):=\mathbb{E}\left(m_{2,0}(X)\right)-\mathbb{E}_{1}(X)^{2}
$$

and

$$
\vee_{2}(X):=\bigvee_{2}(\mu):=\mathbb{E}\left(m_{0,2}(X)\right)-\mathbb{E}_{2}(X)^{2}
$$


The following properties of the modified moments follow from Section 4.

LEMMA 5.2. Let $\mu_{1}, \mu_{2} \in M^{1}\left(\mathbb{N}_{0}^{2}\right)$ having first moments.

(1) $\mathbb{E}_{i}\left(\mu_{1} * \mu_{2}\right)=\mathbb{E}_{i}\left(\mu_{1}\right)+\mathbb{E}_{i}\left(\mu_{1}\right)$ for $i=1,2$.

(2) If the second moments of $\mu_{1}, \mu_{2}$ exist, then, for $i=1,2, \nabla_{i}\left(\mu_{1} * \mu_{2}\right)=$ $\mathbb{V}_{i}\left(\mu_{1}\right)+\mathbb{V}_{i}\left(\mu_{1}\right)$ and $\mathbb{V}_{i}\left(\mu_{1}\right) \geq 0$.

ProOF. Part (1) and the addition formula in (2) follow from Proposition 4.4 (1). The nonnegativity of the modified variance is a consequence of Proposition 4.4 (2) and Jensen's inequality.

We now study random walks on our hypergroup structures on $\mathbb{N}_{0}^{2}$ which are not necessarily homogeneous in time. For this, let $\left(v_{n}\right)_{n \geq 1} \subset M^{1}\left(\mathbb{N}_{0}^{2}\right)$ be a sequence of probability measures. Consider an associated Markov chain $\left(S_{n}\right)_{n \geq 0}$ on $\mathbb{N}_{0}^{2}$ with $S_{0}=(0,0)$ and the transitions

$$
\mathbf{P}\left(S_{n}=(k, l) \mid S_{n-1}=(a, b)\right)=v_{n} * \delta_{(a, b)}(\{(k, l)\}) . \quad\left(n \in \mathbb{N}, k, l, a, b \in \mathbb{N}_{0}\right) .
$$

(Notice that such random walks may be regarded as projections of isotropic random walks on the associated buildings for prime powers $q$.) The following result is an analogue of Kolmogorov's strong law of large numbers.

THEOREM 5.3. Assume that $\left(v_{n}\right)_{n \geq 1}$ and $\left(S_{n}\right)_{n \geq 0}$ are as above with finite second moments. If $\left.\left(r_{n}\right)_{n \in \mathbb{N}} \subset\right] 0, \infty\left[\right.$ satisfies $\lim _{n \rightarrow \infty} r_{n}=\infty$ and

$$
\sum_{n=1}^{\infty} \frac{1}{r_{n}}\left(\mathbb{V}_{1}\left(v_{n}\right)+\mathbb{V}_{2}\left(v_{n}\right)\right)<\infty,
$$

then

$$
\lim _{n \rightarrow \infty} \frac{1}{\sqrt{r_{n}}}\left(S_{n}-\left(\frac{\mathbb{E}_{1}\left(S_{n}\right)+\mathbb{E}_{2}\left(S_{n}\right)}{2}, \frac{\mathbb{E}_{2}\left(S_{n}\right)-\mathbb{E}_{1}\left(S_{n}\right)}{2}\right)\right)=0 \quad \text { a.s. }
$$

PROOF. Proposition 4.4 ensures that the pairs of moment functions $\left(m_{0.1}, m_{0,2}\right)$ and $\left(m_{1.0}, m_{2.0}\right)$ satisfy the conditions of [BH, Theorem 7.3.1] (see also [Z2]). This implies that with probability one,

$$
\lim _{n \rightarrow \infty} \frac{1}{\sqrt{r_{n}}}\left(m_{1.0}\left(S_{n}\right)-\mathbb{E}_{1}\left(S_{n}\right)\right)=0 \quad \text { and } \quad \lim _{n \rightarrow \infty} \frac{1}{\sqrt{r_{n}}}\left(m_{0,1}\left(S_{n}\right)-\mathbb{E}_{2}\left(S_{n}\right)\right)=0 .
$$

On the other hand, Section 4 implies that the functions $m_{0,1}(m, n)-(m+n)$ and $m_{1,0}(m, n)-(m-n)$ are bounded by a constant independent of $m, n$. The theorem now follows after a linear transformation. 
If the random walk is stationary, the above conclusions together with [BH, Theorem 7.3.24] (see also [Z2]) lead to the following strong law.

THEOREM 5.4. Let $\left(S_{n}\right)_{n \geq 0}$ be a stationary random walk on $\mathbb{N}_{0}^{2}$ associated with $v \in$ $M^{1}\left(\mathbb{N}_{0}^{2}\right)$. Assume there exists a constant $\lambda \in\left[1,2\left[\right.\right.$ with $\int_{\mathbb{N}_{0}^{2}}(m+n)^{\lambda} d v(m, n)<\infty$. Then the modified expectations $\mathbb{E}_{1}(\nu)$ and $\mathbb{E}_{2}(v)$ exist, and

$$
\lim _{n \rightarrow \infty} \frac{1}{n^{1 / \lambda}}\left(S_{n}-n\left(\frac{\mathbb{E}_{2}(v)+\mathbb{E}_{1}(v)}{2}, \frac{\mathbb{E}_{2}(v)-\mathbb{E}_{1}(v)}{2}\right)\right)=0 \quad \text { a.s. }
$$

In particular, for $\lambda=1$,

$$
\lim _{n \rightarrow \infty} S_{n} / n=\frac{1}{2}\left(\mathbb{E}_{2}(v)+\mathbb{E}_{1}(v), \mathbb{E}_{2}(v)-\mathbb{E}_{1}(v)\right) .
$$

The following theorem shows that this is no longer true if the first moments of $v$ do not exist.

THEOREM 5.5. Let $\left(S_{n}\right)_{n \geq 0}$ be a homogeneous random walk on $\mathbb{N}_{0}^{2}$ with $\mathbb{E}_{2}\left(S_{1}\right)=\infty$. Then, for all $a>0, \mathbf{P}\left(\left\{S_{n} / n \in[0, a]^{2}\right.\right.$ infinitely often $\left.\}\right)=0$.

For the proof we need the following technical observation.

LEMMA 5.6. There exists a constant $\left.x_{0} \in\right] 0, \ln (q)\left[\right.$ such that for all $x \in\left[0, x_{0}\right]$, $z(x):=1+e^{x} / q+q / e^{x}$ and $m, n \in \mathbb{N}_{0}$,

$$
\begin{aligned}
& C_{m, n}^{q}(z(x), z(x)) \geq C_{m, n+1}^{q}(z(x), z(x)) \quad \text { and } \\
& C_{m, n}^{q}(z(x), z(x)) \geq C_{m+1, n}^{q}(z(x), z(x)) .
\end{aligned}
$$

Proof. By symmetry it suffices to check the second inequality. By Proposition 2.2 (1) and a straightforward computation we have

$$
\begin{aligned}
\frac{C_{m, n}^{q}(z(x), z(x))}{q^{3} K}= & k_{1}^{x, q}\left(\frac{e^{n x}}{q^{2 n+m}}+\frac{e^{m x}}{q^{2 m+n}}\right)+k_{2}^{x, q} \frac{e^{x(m+n)}}{q^{2(m+n)}} \\
& +k_{3}^{x, q}\left(\frac{1}{e^{n x} q^{m}}+\frac{1}{e^{m x} q^{n}}\right)+k_{4}^{x, q} \frac{1}{e^{x(m+n)}}
\end{aligned}
$$

with $K$ defined in (2.5) and with

$$
\begin{array}{rlrl}
k_{1}^{x, q} & :=\frac{\left(e^{x}-1\right)\left(q^{2}-e^{x}\right)\left(e^{2 x}-q\right)}{q\left(q-e^{x}\right)^{2}\left(q^{2}-e^{2 x}\right)}, & k_{2}^{x, q}:=\frac{\left(e^{x}-1\right)^{2}\left(e^{2 x}-q\right)}{\left(q-e^{x}\right)^{2}\left(q^{2}-e^{2 x}\right)} \\
k_{3}^{x, q}:=-\frac{\left(e^{x}-1\right)\left(q^{2}-e^{x}\right)\left(q^{3}-e^{2 x}\right)}{q^{2}\left(q-e^{x}\right)^{2}\left(q^{2}-e^{2 x}\right)}, & k_{4}^{x, q}:=\frac{\left(q^{2}-e^{x}\right)^{2}\left(q^{3}-e^{2 x}\right)}{q^{3}\left(q-e^{x}\right)^{2}\left(q^{2}-e^{2 x}\right)} .
\end{array}
$$


We now regard (5.1) as a function of $m \in \mathbb{R}$. It hence suffices to prove

$$
\left(K q^{3}\right)^{-1} \frac{d}{d m} C_{m, n}^{q}(z(x), z(x))<0
$$

for $m>0$ and $x$ sufficiently small. On the other hand,

$$
e^{(m+n) x}\left(K q^{3}\right)^{-1}(d / d m) C_{m, n}^{q}(z(x), z(x))
$$

is equal to

$$
\begin{aligned}
k_{1}^{x, q}( & \left.-\ln (q)\left(\frac{e^{x}}{q}\right)^{2 n+m}+\ln \left(\frac{e^{x}}{q^{2}}\right)\left(\frac{e^{x}}{q}\right)^{2 m+n}\right)-x k_{4}^{x, q} \\
& +k_{2}^{x, q} \ln \left(\frac{e^{x}}{q^{2}}\right)\left(\frac{e^{x}}{q}\right)^{2(m+n)}-k_{3}^{x, q}\left(\ln (q)\left(\frac{e^{x}}{q}\right)^{m}+x\left(\frac{e^{x}}{q}\right)^{n}\right),
\end{aligned}
$$

where $k_{1}^{x, q}, k_{3}^{x, q}, \ln \left(e^{x} / q^{2}\right) \leq 0$ holds for $x \in[0,(\ln q) / 2]$. Therefore,

$$
\begin{aligned}
\frac{e^{(m+n) x}}{K q^{3}} \frac{d}{d m} C_{m, n}^{q}(z(x), z(x)) \leq & k_{1}^{x, q}\left(-\ln (q)+\ln \left(e^{x} / q^{2}\right)\right)-x k_{4}^{x, q} \\
& +k_{2}^{x, q} \ln \left(e^{x} / q^{2}\right)\left(e^{2 x} / q^{2}\right)^{m+n}-k_{3}^{x, q}(\ln (q)+x)
\end{aligned}
$$

As $\ln \left(e^{x} / q^{2}\right) k_{2}^{x \cdot q}\left(e^{2 x} / q^{2}\right)^{m+n}<0$, the claim follows if we prove that

$$
k_{1}^{x, q}\left(-\ln (q)+\ln \left(e^{x} / q^{2}\right)\right)-x k_{4}^{x, q}-k_{3}^{x, q}(\ln (q)+x)
$$

is negative. For this, we multiply this term by $q^{3}\left(q-e^{x}\right)^{2}\left(q^{2}-e^{2 x}\right)$ and denote this term by $f_{q}(x)$. A simple calculation shows that $f_{q}(0)=0$ with

$$
f_{q}^{\prime}(0):=\left(q^{2}-1\right)(q-1)\left((\ln q) q\left(q^{2}+4 q+1\right)-\left(q^{2}-1\right)\left(1+q+q^{2}\right)\right) .
$$

As $f_{q}^{\prime}(0)<0$ for $q>1$, the claim follows.

PROOF OF THEOREM 5.5. By the Borel-Cantelli lemma it suffices to show that for any $a>0, \sum_{n=1}^{\infty} \mathbf{P}\left(\left\{S_{n} / n . \in[0, a]^{2}\right\}\right)<\infty$. Let $z(\eta)=1+e^{\eta} / q+q / e^{\eta}$ be given as Lemma 5.6. As the $\psi^{0 . m . n}$ defined in Proposition 4.4 (5) are convex, and as $C_{m, n}(z(\eta), \overline{z(\eta)})=\psi^{0, m, n}(\ln (q)-\eta)$, monotone convergence implies

$$
\begin{aligned}
\left.\frac{d}{d \eta} \mathbb{E}\left(C_{S_{1}}^{q}(z(\eta), \overline{z(\eta)})\right)\right|_{\eta=0} & :=\lim _{\eta \downarrow 0} \frac{\mathbb{E}\left(C_{S_{1}}^{q}(z(\eta), \overline{z(\eta)})\right)-\mathbb{E}\left(C_{S_{1}}^{q}(z(0), \overline{z(0)})\right)}{\eta} \\
& =-\lim _{\eta \uparrow \ln q} \mathbb{E}\left(\frac{\psi^{0 . S_{1}}(\ln q)-\psi^{0 . S_{1}}(\eta)}{\ln q-\eta}\right) \\
& =-\mathbb{E}_{2}\left(S_{1}\right)=-\infty
\end{aligned}
$$


As $\left.\left(d e^{-(a+1) \eta} / d \eta\right)\right|_{\eta=0}=-(a+1)$, we find some $\left.\eta_{0} \in\right] 0, \ln q[$ with

$$
\left.e^{(a+1) \eta_{0}} \mathbb{E}\left(C_{S_{1}}^{q}\left(z\left(\eta_{0}\right)\right)\right) \in\right] 0,1[\text {. }
$$

Moreover, Lemma 5.6 together with (3.3) imply

$$
\begin{aligned}
\mathbf{P}\left(\left\{S_{n} / n \in[0, a]^{2}\right\}\right) & \leq \mathbf{P}\left(\left\{C_{S_{n}}^{q}\left(z\left(\eta_{0}\right)\right) \geq C_{\lfloor n a\rfloor+1,\lfloor n a\rfloor+1}^{q}\left(z\left(\eta_{0}\right)\right)\right\}\right) \\
& \leq \mathbf{P}\left(\left\{C_{S_{n}}^{q}\left(z\left(\eta_{0}\right)\right) \geq e^{-2(\lfloor n a\rfloor+1) \eta_{0}}\right\}\right) \\
& \leq e^{2(\{n a\rfloor+1) \eta_{0}} \mathbb{E}\left(C_{S_{n}}^{q}\left(z\left(\eta_{0}\right)\right)\right) \\
& =e^{2(\{n a\rfloor+1) \eta_{0}}\left[\mathbb{E}\left(C_{S_{1}}^{q}\left(z\left(\eta_{0}\right)\right)\right)\right]^{n} \\
& \leq\left[e^{2(a+1) \eta_{0}} \mathbb{E}\left(C_{S_{1}}^{q}\left(z\left(\eta_{0}\right)\right)\right)\right]^{n} .
\end{aligned}
$$

Hence, $\sum_{n=1}^{\infty} \mathbf{P}\left(\left\{S_{n} / n \in[0, a]^{2}\right\}\right)<\infty$, which completes the proof.

\section{A central limit theorem}

We keep the setting of the last section with $q>1$. Let $\left(S_{n}\right)_{n \geq 0}=\left(X_{n}, Y_{n}\right)_{n \geq 0}$ be a stationary random walk on $\mathbb{N}_{0}^{2}$ (with $\mathbb{N}_{0}$-valued components $\left(X_{n}\right)_{n \geq 0}$ and $\left.\left(Y_{n}\right)_{n \geq 0}\right)$ which is associated with some distribution $\nu \in M^{1}\left(\mathbb{N}_{0}^{2}\right)$. We prove the following central limit theorem.

THEOREM 6.1. If $v$ has second moments, then

$$
\frac{1}{\sqrt{n}}\left(X_{n}-n(\tilde{\mu}+\mu) / 2, Y_{n}-n(\tilde{\mu}-\mu) / 2\right)
$$

tends in distribution to the two-dimensional normal distribution $N(0, \Sigma)$ with the positive semidefinite covariance matrix

$$
\Sigma:=\frac{1}{4}\left(\begin{array}{cc}
\sigma_{2}^{2}+2\left(\beta-\mu_{1} \mu_{2}\right)+\sigma_{1}^{2} & \sigma_{2}^{2}-\sigma_{1}^{2} \\
\sigma_{2}^{2}-\sigma_{1}^{2} & \sigma_{2}^{2}-2\left(\beta-\mu_{1} \mu_{2}\right)+\sigma^{2}
\end{array}\right)
$$

with $\mu_{i}:=\mathbb{E}_{i}(\nu), \sigma_{i}^{2}:=\mathbb{V}_{i}(\nu)(i=1,2)$, and $\beta:=\mathbb{E}\left(m_{1.1}(\nu)\right)$.

In the proof we use the notations $C_{m, n}^{q}(z):=C_{m, n}^{q}(z, \bar{z})$ and $\tilde{z}(\xi, \eta):=e^{2 i \xi}+$ $e^{-i \xi}\left(e^{i \eta}+e^{-i \eta}\right)$ from Section 4 . We need the following result.

LEMMA 6.2. For $x, y \in \mathbb{R}$ with $|x|+|y| \rightarrow 0$,

$$
\sup _{m, n \in \mathbb{N}_{0}}\left|C_{m, n}(\tilde{z}(-x, i \ln q-y))-e^{i((m-n) x+(m+n) y)}\right|=O(|x|+|y|) .
$$


PROOF. Put $s_{1}:=e^{-2 i x}, s_{2}:=q e^{i(x+y)}$ and $s_{3}:=e^{i(x-y)} / q$. Then

$$
\tilde{z}(-x, i \ln q-y)=s_{1}+s_{2}+s_{3},
$$

and the assertion follows readily from Proposition 2.2 (1); notice that five of the six summands there have order $O(|x|+|y|)$ independent of $m, n$ for $q>1$, and that the sixth summand

$$
R(m, n):=\frac{K q^{3}}{q^{m+n}} c\left(s_{3}, s_{1}, s_{2}\right) \frac{s_{3}^{m}}{s_{2}^{n}}
$$

satisfies $\sup _{m, n \in \mathbb{N}_{0}}\left|R(m, n)-e^{i((m-n) x+(m+n) y)}\right|=O(|x|+|y|)$.

PROOF OF THEOREM 6.1. We shall prove that for all $x, y \in \mathbb{R}$,

$$
\lim _{n \rightarrow \infty} \mathbb{E}\left(e^{i\left(X_{n}-Y_{n}-n \mu_{1}\right) x / \sqrt{n}+i\left(X_{n}+Y_{n}-n \mu_{2}\right) y / \sqrt{n}}\right)=e^{-\frac{1}{2}(x, y) \bar{\Sigma}(x, y)^{\prime}}
$$

with $\tilde{\Sigma}=\left(\begin{array}{cc}\sigma_{1}^{2} & \beta-\mu_{1} \mu_{2} \\ \beta-\mu_{1} \mu_{2} & \sigma_{2}^{2}\end{array}\right)$. In fact, (6.1) together with Lévy's continuity theorem imply that

$$
\left(X_{n}-Y_{n}-n \mu_{1}, X_{n}+Y_{n}-n \mu_{2}\right) / \sqrt{n}
$$

tends in distribution to $N(0, \tilde{\Sigma})$, and that $\tilde{\Sigma}$ is positive semidefinite. The theorem now follows after a linear transformation.

We now turn to the proof of (6.1). Lemma 6.2 implies that

$$
\lim _{n \rightarrow \infty} \mathbb{E}\left(\left|C_{S_{n}}(z(-x / \sqrt{n}, i \ln q-y / \sqrt{n}))-e^{i\left(\left(X_{n}-Y_{n}\right) x / \sqrt{n}+i\left(X_{n}+Y_{n}\right) y / \sqrt{n}\right)}\right|\right)=0 .
$$

Therefore, it suffices to prove that for all $x, y \in \mathbb{R}$,

$$
\lim _{n \rightarrow \infty} e^{-i\left(\mu_{1} x+\mu_{2} y\right) \sqrt{n}} \mathbb{E}\left(C_{S_{n}}(z(-2 x / \sqrt{n}, i \ln q-y / \sqrt{n}))\right)=e^{-\frac{1}{2}(x, y) \tilde{\Sigma}(x, y)^{\prime}} .
$$

By the multiplicativity of the Fourier transform, this is equivalent to

$$
\lim _{n \rightarrow \infty} e^{-i\left(\mu_{1} x+\mu_{2} y\right) \sqrt{n}} \mathbb{E}\left(C_{S_{1}}(z(-2 x / \sqrt{n}, i \ln q-y / \sqrt{n}))\right)^{n}=e^{-\frac{1}{2}(x, y) \tilde{\Sigma}(x, y)^{\prime}} .
$$

In order to check (6.2), we first notice that for $n \rightarrow \infty$,

$$
e^{-i\left(\mu_{1} x+\mu_{2} y\right) \sqrt{n}}=\left(1-i\left(\mu_{1} x+\mu_{2} y\right) / \sqrt{n}-\left(\mu_{1} x+\mu_{2} y\right)^{2} /(2 n)+O\left(n^{-3 / 2}\right)\right)^{n} .
$$

Moreover, Taylor's formula and Proposition 4.6 imply that

$$
\begin{aligned}
\mathbb{E}\left(C_{S_{1}}\left(z\left(-2 \frac{x}{\sqrt{n}}, i \ln q-\frac{y}{\sqrt{n}}\right)\right)\right)^{n} \\
\quad=\left(1+i \frac{\mu_{1} x+\mu_{2} y}{\sqrt{n}}-\frac{\left(\sigma_{1}^{2}+\mu_{1}^{2}\right) x^{2}+2 \beta x y+\left(\sigma_{2}^{2}+\mu_{2}^{2}\right) y^{2}}{2 n}+o\left(n^{-1}\right)\right)^{n} .
\end{aligned}
$$

Equation (6.2) is now clear, and the proof of the theorem is complete. 
REMARK 6.3. With some additional technical effort, the methods above can be used to derive Berry-Esseen-type orders for the convergence in the central limit theorem. In the case of certain one-dimensional polynomial hypergroups, such qualitative results can be found in [L2] and [V1].

\section{A local central limit theorem}

In this section we derive a local central limit theorem. More precisely, for $v \in$ $M^{1}\left(\mathbb{N}_{0}^{2}\right)$ we search for sequences $\left.\left(c_{n}=c_{n}(v)\right)_{n \in \mathbb{N}} \subset\right] 0, \infty[$ such that

$$
\lim _{n \rightarrow \infty} c_{n} v^{n}(\{(r, s)\})
$$

exists for $(r, s) \in \mathbb{N}_{0}^{2}$ and is positive at least for one $(r, s) \in \mathbb{N}_{0}^{2}$. As $L_{0}=\{(m, n) \in$ $\left.\mathbb{N}_{0}^{2}: m \equiv n \bmod 3\right\}$ is supernormal in $\mathbb{N}_{0}^{2}$, a nontrivial limit above cannot exist for $v \in M^{1}\left(\mathbb{N}_{0}^{2}\right)$ with supp $v \subset L_{i}:=\left\{(m, n) \in \mathbb{N}_{0}^{2}: m-n \equiv i \bmod 3\right\}$ for $i=1,2$. Moreover, the case supp $v \subset L_{0}$ has to be treated separately. Therefore, the conditions of the local limit theorem below are quite natural.

In this section we use the parameterization $z(s, t):=e^{i s}+e^{-i t}+e^{-i(s-t)}(s, t \in$ $[-\pi, \pi])$ for the support $Z_{1}$ of the Plancherel measure on $\widehat{\mathbb{N}}_{0}^{2}$. Notice that $3=z(0,0)$ corresponds to the unique positive character in $Z_{1}$.

THEOREM 7.1. Let $v \in M^{1}\left(\mathbb{N}_{0}^{2}\right)$ with $v \neq \delta_{(0,0)}$. Then the mapping $(s, t) \mapsto$ $\hat{v}(z(s, t))$ is infinitely often differentiable at $(0,0)$ and admits a Taylor expansion with

$$
\hat{v}(z(s, t))=\sigma+\frac{\kappa}{2}\left(s^{2}-s t+t^{2}\right)+O\left((|s|+|t|)^{3}\right) \quad(s, t \rightarrow 0)
$$

with constants $\sigma=\sigma(\nu)=\hat{v}(z(0,0))>0$ and $\kappa=\kappa(\nu)<0$. Moreover, if

$$
\begin{aligned}
C=C(v):= & \frac{q^{11}(q+1)\left(q^{2}+q+1\right)}{(q-1)^{6} 24 \pi^{2}} \int_{-\infty}^{\infty} \int_{-\infty}^{\infty} e^{\frac{x}{2 \sigma}\left(s^{2}-s t+t^{2}\right)} \\
& \times\left(4 s^{6}-12 s^{5} t-3 s^{4} t^{2}+26 s^{3} t^{3}-3 s^{2} t^{4}-12 s t^{5}+4 t^{6}\right) d s d t,
\end{aligned}
$$

then the following holds:

(i) If supp $v$ is not contained in $L_{i}$ for $i=0,1,2$, then for all $(r, s) \in \mathbb{N}_{0}^{2}$,

$$
\lim _{n \rightarrow \infty} \frac{n^{4}}{\sigma^{n}} v^{n}(\{(r, s)\})=C \omega_{\mathbb{N}_{0}^{2}}(\{(r, s)\}) C_{r . s}^{q}(z(0,0), \overline{z(0,0)}) .
$$

(ii) If supp $\nu \subset L_{0}$, then for all $(r, s) \in L_{0}$,

$$
\lim _{n \rightarrow \infty} \frac{n^{4}}{\sigma^{n}} v^{n}(\{(r, s)\})=3 C \omega_{\mathrm{N}_{0}^{2}}(\{(r, s)\}) C_{r, s}^{q}(z(0,0), \overline{z(0,0)}) .
$$


The proof of Theorem 7.1 follows the ideas of the proof of a corresponding local limit theorem for homogeneous trees in [P]. We use the following notation: For a domain $E \subset \mathbb{R}^{2}$ and $f \in C^{(N)}(E)$, put

$$
\left.(s \partial s+t \partial t)^{N} f(s, t)\right|_{\left(s_{0}, \iota_{0}\right)}:=\left.\sum_{k=0}^{N}\left(\begin{array}{l}
N \\
k
\end{array}\right) s^{k} t^{N-k} \frac{\partial^{N}}{\partial s^{k} \partial t^{N-k}} f(s, t)\right|_{\left(s_{0}, t_{0}\right)} .
$$

The proof of Theorem 7.1 will be decomposed into the following steps:

(A) Find a finite set $S \subset Z_{1}$ with $3 \in S$ such that for all $z \in Z_{1} \backslash S,|\hat{v}(z)|<\hat{v}$ (3).

(B) In a neighbourhood $U \subset[-\pi, \pi]^{2}$ of $(0,0)$ one has the Taylor expansion

$$
\hat{v}(z(s, t))=\hat{v}(3)+\left.(s \partial s+t \partial t)^{2} \hat{v}(z(s, t))\right|_{(0,0)}+O\left((|s|+|t|)^{3}\right)
$$

(C) Apply the inversion formula for the Fourier transform on $\mathbb{N}_{0}^{2}$.

Step (A) is decomposed in some lemmata. The first one is obvious.

LEMMA 7.2. Let $n \in \mathbb{N}$ and $z_{1}, \ldots, z_{n} \in \mathbb{C}$. Then $\left|\sum_{i=1}^{n} z_{i}\right|=\sum_{i=1}^{n}\left|z_{i}\right|$ if and only if $\arg \left(z_{i}\right)=\arg \left(z_{j}\right)$ for all $i, j \in\{1, \ldots, n\}$.

LEMMA 7.3. Let $v \in M^{1}\left(\mathbb{N}_{0}^{2}\right)$ with $v \neq \delta_{0,0}$. Then, for all $z \in Z_{1} \backslash\left\{3 e^{2 k \pi i / 3}: k=\right.$ $0,1,2\},|\hat{v}(z)|<\hat{v}(3)$. Moreover, if supp $v \not \subset L_{i}$ for $i=0,1,2$, then $\left|\hat{v}\left(3 e^{ \pm 2 \pi i / 3}\right)\right|<$ $\hat{v}(3)$. Otherwise, $\left|\hat{v}\left(3 e^{ \pm 2 \pi i / 3}\right)\right|=\hat{v}(3)$.

ProOF. Let $z \in Z_{1} \backslash\left\{3 e^{2 k \pi i / 3}: k=0,1,2\right\}$. We prove that

$$
\left|C_{m, n}^{q}(z, \bar{z})\right|<C_{m, n}^{q}(3,3) \quad \text { for } \quad(m, n) \neq(0,0) .
$$

The first statement is then clear. To prove (7.2) for $(m, n) \neq(0,0)$, consider the $T_{i, j}$ appearing in the expansion of Proposition 3.3. If $(m, n) \in L_{0}$ then, by Lemma $3.7(1)$, $T_{1,1}$ appears. Therefore, as $\left|T_{m, n}(z, \bar{z})\right| \leq T_{m, n}(3,3)$ for $m, n \in \mathbb{N}_{0}$ and $z \in Z_{1}$, it suffices to check

$$
\left|T_{1,1}(z, \bar{z})\right|<T_{1.1}(3,3)
$$

According to Section 2, we write $z=z(s, t)=e^{i s}+e^{-i t}+e^{-i(s-t)}$ with $s, t \in[0,2 \pi]$. As $T_{1,1}(z(s, t), \overline{z(s, t)})$ is equal to

$$
e^{-i(s-2 t)}+e^{i(s-2 t)}+e^{i(2 s-t)}+e^{i(-2 s+t)}+e^{-i(s+t)}+e^{i(s+t)},
$$

and as $\left|T_{1.1}(z(s, t), \overline{z(s, t)})\right|=T_{1,1}(z(0,0), \overline{z(0,0)})=6$, Lemma 7.2 implies that $s, t \in 2 \pi / 3 \cdot \mathbb{Z}$ and $s+t \in \pi \cdot \mathbb{Z}$. Hence, $s=2 \pi-t=k \pi / 3$ for $k \in\{0,1,2\}$, that is, we obtain the three exceptional points, and (7.3) and thus (7.2) are clear. If $(m, n) \in L_{1}$, 
then by Lemma $3.7(2), T_{1,0}$ appears with a positive coefficient, and it suffices to check $\left|T_{1,0}(z, \bar{z})\right|<T_{1,0}(3,3)$. As $T_{1,0}(z(s, t), \overline{z(s, t)})=2\left(e^{i s}+e^{-i t}+e^{i(t-s)}\right)$, we again obtain the three exceptional points above which proves (7.2) in this case. The case $(m, n) \in L_{2}$ follows by symmetry. This completes the proof of the first statement.

Now consider $v$ with supp $v \not \subset L_{i}$ for $i=0,1$ or 2 . Then take $\left(m_{1}, n_{1}\right)$ and $\left(m_{2}, n_{2}\right)$ from supp $v$ with $\left(m_{1}-n_{1}\right) \not \equiv\left(m_{2}-n_{2}\right)$ mod 3. Remark 2.8 (2) and Lemma 7.2 then yield for $z:=3 e^{2 / 3 \pi i}$ that

$$
\begin{aligned}
& \left|C_{m_{1}, n_{1}}^{q}(z, \bar{z}) v\left(\left\{\left(m_{1}, n_{1}\right)\right\}\right)+C_{m_{2}, n_{2}}^{q}(z, \bar{z}) v\left(\left\{\left(m_{2}, n_{2}\right)\right\}\right)\right| \\
& \quad<C_{m_{1}, n_{1}}^{q}(3,3) v\left(\left\{\left(m_{1}, n_{1}\right)\right\}\right)+C_{m_{2}, n_{2}}^{q}(3,3) v\left(\left\{\left(m_{2}, n_{2}\right)\right\}\right) .
\end{aligned}
$$

As $\left|C_{m, n}^{q}(z, \bar{z})\right| \leq\left|C_{m, n}^{q}(3,3)\right|$ for all $z \in Z_{1}$ and $m, n \in \mathbb{N}_{0}$, the second statement is clear. The final statement follows from Remark 2.8 (2).

We next turn to Step (B) of the proof.

LEMMA 7.4. For each $N \in \mathbb{N}_{0}$ there exists a constant $K_{N}>0$ such that for all $m, n, k, l \in \mathbb{N}_{0}$ with $k+l \leq N$ and all $s, t \in[0,2 \pi]$

$$
\left|\frac{\partial^{k+l}}{\partial s^{k} \partial t^{t}} C_{m, n}^{q}(z(s, t), \overline{z(s, t)})\right| \leq K_{N} .
$$

Moreover,

$$
\left.\frac{\partial^{k+l}}{\partial s^{k} \partial t^{l}} C_{m, n}^{q}(z(s, t), \overline{z(s, t)})\right|_{(0,0)}=0 \text { for } k+l=1 .
$$

PROOF. Remark 3.6 (4) implies that

$$
\frac{\partial^{k+l}}{\partial s^{k} \partial t^{l}} C_{m, n}^{q}(z(s, t), \overline{z(s, t)})=\sum_{i, j} h_{m, n, i, j} \frac{\partial^{k+l}}{\partial s^{k} \partial t^{l}} T_{i, j}(z(s, t), \overline{z(s, t)})
$$

with $\sum_{i, j} h_{m, n, i, j} \leq Q^{q}(m, n) / q^{m+n}$ where $Q^{q}$ is a polynomial. The definition of the $T_{i, j}$ shows that

$$
\left|\frac{\partial^{k+l}}{\partial s^{k} \partial t^{l}} T_{i, j}(z(s, t), \overline{z(s, t)})\right| \leq 62^{k+l}(i+j)^{k+l} .
$$

Hence,

$$
\left|\frac{\partial^{k+l}}{\partial s^{k} \partial t^{l}} C_{m, n}^{q}(z(s, t), \overline{z(s, t)})\right| \leq 62^{k+l} \frac{Q^{q}(m, n)}{q^{m+n}} .
$$

As $q>1$, the first part of the lemma follows. Finally, as

$$
\left.\frac{\partial}{\partial s} T_{i, j}(z(s, t), \overline{z(s, t)})\right|_{(0,0)}=\left.\frac{\partial}{\partial t} T_{i, j}(z(s, t), \overline{z(s, t)})\right|_{(0,0)}=0,
$$

the second assertion is also clear. 
We are now ready to derive the following Taylor formula.

Proposition 7.5. Let $v \in M^{1}\left(\mathbb{N}_{0}^{2}\right)$ with $v \neq \delta_{0,0}$. Then $\sigma:=\hat{v}(z(0,0))>0$, and there exists $\kappa<0$ such that

$$
\hat{v}(z(s, t))=\sigma+\frac{\kappa}{2}\left(s^{2}-s t+t^{2}\right)+O\left((|s|+|t|)^{3}\right) \quad \text { for } s, t \rightarrow 0 .
$$

PROOF. Remark 3.6 (2) yields $\sigma>0$, and by Lemma 7.4 there is a constant $K>0$ such that for all $m, n, k, l \in \mathbb{N}_{0}$ with $k+l \leq 3$,

$$
\left|\frac{\partial^{k+l}}{\partial s^{k} \partial t^{l}} \bar{C}_{m, n}^{q}(z(s, t), \overline{z(s, t)})\right| \leq K .
$$

Dominated convergence hence implies that the derivatives

$$
\frac{\partial^{k+l}}{\partial s^{k} \partial t^{l}} \hat{v}(z(s, t))
$$

exist for $k+l \leq 3$ with

$$
\frac{\partial^{k+l}}{\partial s^{k} \partial t^{l}} \hat{v}(z(s, t))=\int_{\mathbb{N}_{0}^{2}} \frac{\partial^{k+l}}{\partial s^{k} \partial t^{l}} \bar{C}_{m, n}^{q}(z(s, t), \overline{z(s, t)}) d v(m, n) .
$$

As Lemma 7.4 leads to

$$
\left.\frac{\partial}{\partial s} \hat{v}(z(s, t))\right|_{(0,0)}=\left.\frac{\partial}{\partial t} \hat{v}(z(s, t))\right|_{(0,0)}=0
$$

we obtain

$$
\hat{v}(z(s, t))=\hat{v}(z(0,0))+\left.(s \partial s+t \partial d)^{2} \hat{v}(z(s, t))\right|_{(0,0)}+O\left((|s|+|t|)^{3}\right) .
$$

A simple calculation gives for $T_{i, j}(z):=T_{i, j}(z, \bar{z})$

and

$$
\left.\frac{\partial^{2}}{\partial s^{2}} T_{i, j}(z(s, t))\right|_{(0,0)}=-4\left(i^{2}+i j+j^{2}\right)=\left.\frac{\partial^{2}}{\partial t^{2}} T_{i, j}(z(s, t))\right|_{(0,0)}
$$

$$
\left.\frac{\partial^{2}}{\partial s \partial t} T_{i, j}(z(s, t))\right|_{(0,0)}=2\left(i^{2}+i j+j^{2}\right) .
$$

Hence, by Proposition 3.3,

$$
\kappa_{m, n}:=\left.\frac{\partial^{2}}{\partial s^{2}} C_{m, n}^{q}(z(s, t), \overline{z(s, t)})\right|_{(0,0)}<0
$$


with

$$
\left.\frac{\partial^{2}}{\partial t^{2}} C_{m, n}^{q}(z(s, t), \overline{z(s, t)})\right|_{(0,0)}=\kappa_{m, n}=-\left.2 \frac{\partial^{2}}{\partial s \partial t} C_{m, n}^{q}(z(s, t), \overline{z(s, t)})\right|_{(0,0)} .
$$

Therefore, as $v \neq \delta_{0,0}, \kappa:=\int_{\mathbb{N}_{0}^{2}} \kappa_{n, m} d v(m, n)<0$ with

$$
\kappa=\left.\frac{\partial^{2}}{\partial s^{2}} \hat{v}(z(s, t))\right|_{(0,0)}=\left.\frac{\partial^{2}}{\partial t^{2}} \hat{v}(z(s, t))\right|_{(0,0)}=-\left.2 \frac{\partial^{2}}{\partial s \partial t} \hat{v}(z(s, t))\right|_{(0,0)} .
$$

The lemma now follows from the Taylor expansion above.

We now turn to Step (C) of the proof.

LEMMA 7.6. Let $v \in M^{1}\left(\mathbb{N}_{0}^{2}\right), n \in \mathbb{N}$, and let the function $c$ be given as in (2.6). Then the $n$-fold convolution power of $v$ is given by

$$
\begin{aligned}
v^{n}(\{(r, l)\})= & \frac{q^{5}(q+1)\left(q^{2}+q+1\right)}{24 \pi^{2}} \omega(\{(r, l)\}) \int_{-\pi}^{\pi} \int_{-\pi}^{\pi} \hat{v}^{n}(z(s, t)) \\
& \times C_{r, l}(z(s, t), \overline{z(s, t)}) \frac{d s d t}{\left|k\left(e^{i s}, e^{-i t}, e^{-i(s-t)}\right)\right|^{2}}
\end{aligned}
$$

for $r, l \in \mathbb{N}_{0}$, where $\omega$ denotes the Haar measure.

PROOF. The inversion formula (see [BH, Theorem 2.2.36]) yields

$$
v^{n}(\{(r, l)\})=\omega(\{(r, l)\}) \int_{Z_{l}} \hat{v}^{n}(x+i y) C_{r, l}^{q}(x+i y, x-i y) R(x+i y) d(x, y)
$$

with $R$ as in Theorem 2.4. The transformation

$$
x=\cos (s)+\cos (t)+\cos (s-t), \quad y=\sin (s)-\sin (t)-\sin (s-t),
$$

and a short computation as in [CM, Section 5] now yield the lemma.

We now complete the proof of Theorem 7.1. Let $v, \kappa, \sigma$, and $C$ be given as there where $C<\infty$ obviously holds. Let $n \in \mathbb{N}$ and $l, r \in \mathbb{N}_{0}$. Consider the points $\left(s_{1}, t_{1}\right)=(0,0),\left(s_{2}, t_{2}\right)=(2 \pi / 3,-2 \pi / 3)$ and $\left(s_{3}, t_{3}\right)=(-2 \pi / 3,2 \pi / 3)$. For $\epsilon>0$ and $i=1,2,3$, let

$$
U_{i, \epsilon}:=\left\{(s, t) \in[-\pi, \pi]^{2}:\left|s-s_{i}\right|<\epsilon,\left|t-t_{i}\right|<\epsilon\right\}
$$

and

$$
U_{\epsilon}:=[-\pi, \pi]^{2} \backslash\left(U_{1, \epsilon} \cup U_{2, \epsilon} \cup U_{3, \epsilon}\right)
$$


If $w(s, t):=\left|k\left(e^{i s}, e^{-i t}, e^{-i(s-t)}\right)\right|^{-2}$, the integral in Lemma 7.6 is given by

$$
\begin{aligned}
& \int_{-\pi}^{\pi} \int_{-\pi}^{\pi} \hat{v}^{n}(z(s, t)) C_{r, l}^{q}(z(s, t), \overline{z(s, t)}) w(s, t) d s d t \\
& \quad=\left(\sum_{i=1}^{3} \int_{U_{i, t}}+\int_{U_{\epsilon}}\right) \hat{v}^{n}(z(s, t)) C_{r, l}^{q}(z(s, t), \overline{z(s, t)}) w(s, t) d(s, t) \\
& =: I_{1}(n)+I_{2}(n)+I_{3}(n)+I_{4}(n) .
\end{aligned}
$$

We now check Part (1) of the theorem. Assume that supp $v$ is not contained all $L_{i}$. According to Lemma 7.3 there is a constant $\eta_{1}<\gamma$ with $\hat{v}(z(s, t)) \leq \eta_{1}$ for all $(s, t) \in U_{\epsilon}$. Hence, $\left|I_{4}(n)\right| \leq \eta^{n}$ and $\lim _{n \rightarrow \infty} n^{4} \sigma^{-n} I_{4}(n)=0$. Lemma 7.3 also implies

$$
\lim _{n \rightarrow \infty} \frac{n^{4}}{\sigma^{n}} I_{3}(n)=\lim _{n \rightarrow \infty} \frac{n^{4}}{\sigma^{n}} I_{2}(n)=0
$$

In order to handle $I_{1}(n)$, we observe that $s^{2}-s t+t^{2} \geq 0$ and conclude that we find an $\epsilon>0$ such that for $\kappa<0$ as in Proposition 7.5 and all $|s|+|t|<\epsilon$,

$$
\begin{aligned}
1+\frac{\kappa}{2 \sigma}\left(s^{2}-s t+t^{2}\right)+O\left((|s|+|t|)^{3}\right) & \leq 1+\frac{\kappa}{4 \sigma}\left(s^{2}-s t+t^{2}\right) \\
& \leq e^{\frac{\kappa}{4 \sigma}\left(s^{2}-s t+t^{2}\right)} .
\end{aligned}
$$

Hence, by Proposition 7.5,

$$
\left(\frac{\hat{v}(z(s / \sqrt{n}, t / \sqrt{n}))}{\sigma}\right)^{n} \leq e^{\frac{\kappa}{\alpha(}\left(s^{2}-s t+t^{2}\right)} .
$$

Moreover, the function $w$ above is $C^{\infty}$, and a computation shows that

$$
\left.\frac{\partial^{k+l}}{\partial s^{k} \partial t^{l}} w(s, t)\right|_{(0,0)}=0 \quad \text { for } k+l \leq 5,
$$

and that $g(s, t):=\left.(6 !)^{-1}(s \partial s+t \partial t)^{6} w(s, t)\right|_{(0,0)}$ satisfies

(7.6) $g(s, t)=\left(\frac{q}{q-1}\right)^{6}\left(4 s^{6}-12 s^{5} t-3 s^{4} t^{2}+26 s^{3} t^{3}-3 s^{2} t^{4}-12 s t^{5}+4 t^{6}\right)$.

A Taylor expansion at $(0,0)$ thus implies

$$
w(s, t)=g(s, t)+O\left((|s|+|t|)^{7}\right) .
$$


Using the transformation $s \mapsto s / \sqrt{n}$ and $t \mapsto t / \sqrt{n}$ and the abbreviation $C_{r, l}^{q}(z):=$ $C_{r, l}^{q}(z, \bar{z})$, we may write

$$
\begin{aligned}
\sigma^{-n} n^{4} I_{1}(n) & =n^{3} \int_{-n \epsilon}^{n \epsilon} \int_{-n \epsilon}^{n \epsilon} h_{n}(s, t) C_{r, l}^{q}\left(z\left(\frac{s}{\sqrt{n}}, \frac{t}{\sqrt{n}}\right)\right) w\left(\frac{s}{\sqrt{n}}, \frac{t}{\sqrt{n}}\right) d s d t \\
& =\int_{-\infty}^{\infty} \int_{-\infty}^{\infty} h_{n}(s, t) g_{n}(s, t) C_{r, l}^{q}\left(z\left(\frac{s}{\sqrt{n}}, \frac{t}{\sqrt{n}}\right)\right) d s d t
\end{aligned}
$$

with

$$
\begin{aligned}
& h_{n}(s, t):=\mathbf{1}_{[-n \epsilon, n \epsilon]^{2}}\left(\frac{\hat{v}(z(s / \sqrt{n}, t / \sqrt{n}))}{\sigma}\right)^{n}, \\
& g_{n}(s, t):=n^{3} \mathbf{1}_{[-n \epsilon, n \epsilon]^{2}} w\left(\frac{s}{\sqrt{n}}, \frac{t}{\sqrt{n}}\right) .
\end{aligned}
$$

Equation (7.5) yields that

$$
\begin{aligned}
\left|h_{n}(s, t)\right| & =\left|\mathbf{1}_{[-n \epsilon, n \epsilon]^{2}}\left(1+\frac{\kappa}{2 \sigma} \frac{s^{2}-s t+t^{2}}{n}+(|s|+|t|)^{3} O\left(n^{-3 / 2}\right)\right)^{n}\right| \\
& \leq e^{\frac{k}{\sigma \sigma}\left(s^{2}-s t+t^{2}\right)}
\end{aligned}
$$

and $\lim _{n \rightarrow \infty} h_{n}(s, t)=e^{\frac{x}{2 \sigma}\left(s^{2}-s t / 2+t^{2}\right)}=: h(s, t)$. Moreover, (7.6) and (7.7) imply that there is a constant $\widetilde{K}>0$ with

$$
\begin{aligned}
\left|g_{n}(s, t)\right| & =\left|\mathbf{1}_{[-n \epsilon, n \epsilon]^{2}}\left(\left.\frac{1}{6 !}(s \partial s+t \partial t)^{6} g(s, t)\right|_{(0,0)}+(|s|+|t|)^{7} O\left(n^{-1 / 2}\right)\right)\right| \\
& \leq \frac{\widetilde{K}}{6 !}(s+t)^{6} .
\end{aligned}
$$

Therefore, $\int_{-\infty}^{\infty} \int_{-\infty}^{\infty} e^{(\kappa / 4 \sigma)\left(s^{2}-s t+t^{2}\right)}(s+t)^{6} d s d t<\infty$ and the dominated convergence theorem lead to

$$
\lim _{n \rightarrow \infty} \sigma^{-n} n^{4} I_{1}(n)=C_{r, l}^{q}(z(0,0)) \int_{-\infty}^{\infty} \int_{-\infty}^{\infty} h(s, t) g(s, t) d s d t .
$$

This implies the first part of the theorem.

Now assume that $(r, l) \in L_{0}=$ and $\operatorname{supp} v \subset L_{0}$. In this case, the arguments at the end of the proof of Lemma 7.3 show that

$$
\begin{aligned}
& \hat{v}^{n}(z(-2 / 3 \pi+s, 2 / 3 \pi+t)) C_{r, l}^{q}(z(-2 / 3 \pi+s, 2 / 3 \pi+t)) \\
& \quad=\hat{v}^{n}(z(s, t)) C_{r, l}^{q}(z(s, t)) .
\end{aligned}
$$

Moreover, $w(-2 / 3 \pi+s, 2 / 3 \pi+t)=w(s, t)$ implies $I_{2}(n)=I_{1}(n)$. In a similar way, we have $I_{3}(n)=I_{1}(n)$. As the limits for $I_{1}(n)$ and $I_{4}(n)$ are the same as in the previous case, the second part of Theorem 7.1 follows readily. 


\section{References}

[AW] R. Askey and J. A. Wilson, Some basic hypergeometric orthogonal polynomials that generalize Jacobi polynomials, Mem. Amer. Math. Soc. 54 (Amer. Math. Soc., Providence, RI, 1985).

[BH] W. R. Bloom and H. Heyer, Harmonic analysis of probability measures on hypergroups (de Gruyter, Berlin, 1995).

[BG] M. Bouhaik and L. Gallardo, 'Un théorème limit central dans un hypergroup bidimensionnel', Ann. Inst. H. Poincaré Probab. Statist. 28 (1992), 47-61.

[B] K. S. Brown, Buildings (Springer, New York, 1989).

[C] D. I. Cartwright, 'Spherical harmonic analysis on buildings of type $\tilde{A}_{n}$ ', Monatsh. Math. 133 (2001), 93-109.

[CMSZ] D. I. Cartwright, A. M. Mantero, T. Steger and A. Zappa, 'Groups acting simply transitively on the vertices of a building of type $\widetilde{A_{2}}$. I, II', Geom. Dedicata 47 (1993), 143-166 and $167-223$.

[CM] D. I. Cartwright and W. Młotkowski, 'Harmonic analysis for groups acting on triangle buildings', J. Austral. Math. Soc. Ser. A 56 (1994), 345-383.

[CMS] D. I. Cartwright, W. Młotkowski and T. Steger, 'Property (T) and $\tilde{A_{2}}$ groups', Ann. Inst. Fourier (Grenoble) 44 (1994), 213-248.

[CW] D. I. Cartwright and W. Woess, 'Isotropic random walks in a building of type $\tilde{a}_{d}$ ', Preprint, 2001.

[F] W. Feller, An introduction to probability theory and its applications, volume II, 2nd edition (Wiley, New York, 1971).

[Kw] T. H. Koornwinder, 'Orthogonal polynomials in two variables which are eigenfunctions of two algebraically independent partial differential operators III, IV', Indag. Math. 36 (1974), 357-369 and 370-381.

[Ky] A. Korányi (editor), Harmonic functions on trees and buildings, Contemp. Math. 206, (Amer. Math. Soc., Providence, RI, 1997).

[LR] R. Lasser and M. Rösler, 'A note on property (T) of orthogonal polynomials', Arch. Math. 60 (1993), 459-463.

[L1] M. Lindlbauer, Grenzwertsätze für Irrfahrten auf Dreiecksgebäuden und den assoziierten polynomiellen Hypergruppen (Ph.D. Thesis, Universität Tübingen, 1998).

[L2] - 'On the rate of convergence of the laws of Markov chains associated with orthogonal polynomials', J. Comp. Appl. Math. 99 (1998), 287-297.

[Mac1] I. G. Macdonald, Spherical functions on a group of p-adic type, Publications of the Ramanujan Institute, No. 2 (Ramanujan Institute, University of Madras, Madras, 1971).

[Mac2] - Symmetric functions and Hall polynomials, 2nd edition (Oxford University Press, New York, 1995).

[M] F. Miller Maley, 'The Hall polynomial revisited', J. Algebra 184 (1996), 363-371.

[MZ] A. M. Mantero and A. Zappa, 'Spherical functions and spectrum of the Laplace operators on buildings of rank 2', Boll. Un. Mat. Ital. B (7) 8 (1994), 419-475.

[P] M. A. Picardello, 'Spherical functions and local limit theorems on free groups', Ann. Math. Pura Appl. (4) 33 (1983), 177-191.

[R] M. Ronan, Lectures on buildings (Academic Press, Boston, 1989).

[Sa] S. Sawyer, 'Isotropic random walks in a tree', Z. Wahrsch. Verw. Gebiete 42 (1978), 279-292.

[Si] B. Simon, Representations of finite and compact groups (Amer. Math. Soc., 1996).

[T] J. Tits, 'Spheres of radius 2 in triangle buildings I', in: Finite geometries, buildings and related topics (ed. W. Kantor et al.) (Clarendon Press, Oxford, 1990) pp. 17-28.

[V1] M. Voit, 'Central limit theorems for random walks on $\mathbb{N}_{0}$ that are associated with orthogonal 
polynomials', J. Multivar. Anal. 34 (1990), 290-322.

[V2] _ - 'Laws of large numbers for polynomial hypergroups and some applications', $J$. Theor. Probab. 3 (1990), 245-266.

[V3] _ _ 'A law of the iterated logarithm for Markov chains on $\mathbb{N}_{0}$ associated with orthogonal polynomials', J. Theor. Probab. 6 (1993), 653-669.

[Z1] H. Zeuner, 'Laws of large numbers for hypergroups on $\mathbb{R}_{+}$', Math. Ann. 283(1989), 657-678.

[Z2] _ _Moment functions and laws of large numbers on hypergroups', Math. Z. 211 (1992), 369-407.

[Z3] _ 'Polynomial hypergroups in several variables', Arch. Math. 58 (1992), 425-434.

Mathematisches Institut

Universität Tübingen

Auf der Morgenstelle 10

72076 Tübingen

Germany

and

GSF-Forschungszentrum

für Umwelt und Gesundheit

85764 Neuherberg

Germany
Mathematisches Institut

Universität Tübingen

Auf der Morgenstelle 10

72076 Tübingen

Germany

e-mail: michael.voit@uni-tuebingen.de 
